\title{
Interdependence of Multiple Theta Generators in the Hippocampus: a Partial Coherence Analysis
}

\author{
Bernat Kocsis, ${ }^{1,3}$ Anatol Bragin, ${ }^{2}$ and György Buzsáki ${ }^{2}$ \\ 1 Laboratory of Neurophysiology, Department of Psychiatry, Harvard Medical School, Boston, Massachusetts 02115, \\ 2 Center for Molecular and Behavioral Neuroscience, Rutgers, The State University of New Jersey, Newark, New Jersey \\ 07102, and ${ }^{3}$ National Institute of Neurosurgery, Budapest, Hungary
}

The extracellularly recorded theta oscillation reflects a dynamic interaction of various synaptic and cellular mechanisms. Because the spatially overlapping dipoles responsible for the generation of theta field oscillation may represent different mechanisms, their separation might provide clues with regard to their origin and significance. We used a novel approach, partial coherence analysis, to reveal the various components of the theta rhythm and the relationship among its generators. Hippocampal field activity was recorded by a 16-site silicon probe in the CA1-dentate gyrus axis of the awake rat. Field patterns, recorded from various intrahippocampal or entorhinal cortex sites, were used to remove activity caused by a common source by the partialization procedure. The findings revealed highly coherent coupling between theta signals recorded (1) from the hippocampal fissure and stratum (str.) oriens of the CA1 region and (2) between CA1 stratum radiatum and the dentate molecular layer. The results of partial coherence analysis indicated that rhythmic input from the entorhinal cortex explained theta coherence between signals recorded from the hippocampal fissure and str. oriens but not the coherence between signals derived from str. radiatum and the dentate molecular layer. After bilateral lesions of the entorhinal cortex, all signals recorded from both below and above the CA1 hippocampal pyramidal cell layer became highly coherent. These observations indicate the presence of two, relatively independent, theta generators in the hippocampus, which are mediated by the entorhinal cortex and the CA3-mossy cell recurrent circuitry, respectively. The CA3-mossy cell theta generator is partially suppressed by the dentate gyrus interneuronal output in the intact brain. We suggest that timing of the action potentials of pyramidal cells during the theta cycle is determined by the cooperation between the active CA3 neurons and the entorhinal input.

Key words: hippocampus; theta rhythm; CA3 recurrent system; phase-locking; coding; neuronal oscillators; partial coherence
The rat hippocampal formation exhibits rhythmic oscillatory field potentials at theta $(5-10 \mathrm{~Hz})$ frequency during its activated, exploration-associated state and rapid eye movement (REM) sleep (Vanderwolf, 1969). Theta oscillation has been associated with a variety of different overt and covert behaviors (cf. Buzsáki et al., 1983; Stewart and Fox, 1990; Bland and Colom, 1993b; Vinogradova, 1995; Vertes and Kocsis, 1997). Furthermore, the timing of "place cell" spikes during the theta cycle may precisely indicate the spatial location of the animal (O'Keefe and Recce, 1993; Skaggs and McNaughton, 1996). The phase-locked discharge of the concurrently active pyramidal and granule cells may be critical for providing sufficient depolarization of their postsynaptic targets and, consequently, for the induction of synaptic plasticity (Larson and Lynch, 1986; Huerta and Lisman, 1996; Holscher et al., 1997).

Theta oscillation reflects a consortium of mechanisms at the

\footnotetext{
Received Feb. 10, 1999; revised April 5, 1999; accepted May 6, 1999.

This work was supported by National Institutes of Health Grants NS34994, MH54671, and 1P41RR09754, Hungarian Scientific Research Fund (OTKA) Grant T-17778, and Hungarian Ministry of Health Grant ETT-085/1996-04. We thank Allan Hobson, Robert Stickgold, Gerard Gebber, Maciej Kaminski, Hajime Hirase, and Ken Harris for their comments on this manuscript, and Jamie Hetke and Ken Wise for supplying us silicon probes.

Correspondence should be addressed to Bernat Kocsis, Laboratory of Neurophysiology, Department of Psychiatry, Harvard Medical School, 74 Fenwood Road, Boston, MA 02115. E-mail: bkocsis@hms.harvard.edu.

Dr. Bragin's present address: Department of Neurology, University of California at Los Angeles School of Medicine, 710 Westwood Plaza, Los Angeles, CA 90095. Copyright (C) 1999 Society for Neuroscience $0270-6474 / 99 / 196200-13 \$ 05.00 / 0$
}

cellular, local circuit, and neural systems levels. It is enhanced by intrinsic, voltage-dependent membrane oscillations of pyramidal cells (Leung and Yim, 1991; Kamondi et al., 1998a) and by the phase-related discharge of hippocampopetal neurons in many structures of the brain (Kocsis and Vertes, 1994, 1997; Vertes and Kocsis, 1997). Of these afferents, the septal input and the entorhinal afferents appear most critical (Petsche et al., 1962; Alonso and Garcia-Austt, 1987; Stewart and Fox, 1990; Lee et al., 1994).

The rhythmic somatic inhibition of pyramidal cells during theta oscillation is well coordinated by concurrent excitation of their dendrites (Kamondi et al., 1998b). The overall effect is that the majority of principal cells remain silent, and the action potentials of the remaining minority are phase-locked to the quantal periods of theta and gamma frequencies (Bragin et al., 1995). Because both interneurons and principal cells are phase-locked to the field oscillation, one might expect that intrahippocampal association and commissural pathways contribute significantly to the extracellular currents underlying theta. Indeed, theta-like field oscillations can also be induced in the hippocampal slice preparation in vitro, and this rhythmic pattern depends on the integrity of the CA3 region in vitro (Konopacki et al., 1987, 1988; MacVicar and Tse, 1989; Traub et al., 1992; Bland and Colom, 1993a; Huerta and Lisman, 1993, 1996; Williams and Kauer, 1997; Fisahn et al., 1998; McMahon et al., 1998). In contrast to the in vitro observations, previous current source density studies in vivo indicated that the contribution of the associational and commissural afferents is rather small compared with other afferents (Buzsáki et al., 
1986; Brankack et al., 1993). This may be so because only an estimated $1 \%$ of CA3 pyramidal cells are active during the theta cycle (Buzsáki, 1989; Barnes, 1990). Nevertheless, the issue of the contribution of the intrahippocampal associational system to theta generation may be important because the cooperative activity of the direct entorhinal inputs and associational afferents may be critical for the discharge of pyramidal neurons in the intact brain (Hasselmo and Bower, 1993; Buzsáki et al., 1995; Wallenstein et al., 1998).

Because the spatially overlapping dipoles responsible for the generation of theta field oscillation may represent different mechanisms, their separation might provide clues with regard to their origin and significance. In the present study, we used a novel approach, partial coherence analysis, to identify the various components of theta rhythm and the relationship among its generators.

\section{MATERIALS AND METHODS}

Surgery and recording. The subjects of the present study were the same as those used for analyzing gamma activity in vivo (Bragin et al., 1995). Briefly, Sprague Dawley rats $(300-450 \mathrm{gm})$ were anesthetized with a mixture $(4 \mathrm{ml} / \mathrm{kg})$ of ketamine $(25 \mathrm{mg} / \mathrm{ml})$, xylazine $(1.3 \mathrm{mg} / \mathrm{ml})$, and acepromazine $(0.25 \mathrm{mg} / \mathrm{ml})$. Pairs of stainless steel wires $(100 \mu \mathrm{m}$ in diameter) with $0.5 \mathrm{~mm}$ vertical tip separation were placed in the angular bundle unilaterally (right side) or bilaterally to stimulate the medial perforant path afferents to the hippocampus [anteroposterior (AP), -7.0 $\mathrm{mm}$ from bregma; lateral (L), $3.5 \mathrm{~mm}$ from midline; ventral (V), -3.0 $\mathrm{mm})$. Another electrode pair was placed into the ventral hippocampal commissure (AP, $-0.8 ; \mathrm{L}, 0.5 ; \mathrm{V},-4.2)$ to stimulate the commissural afferents to the CA1-CA3 regions and the dentate gyrus. For simultaneous recording of field potentials and unit activity in different hippocampal regions and layers, silicon probes micromachined with thinfilm technology were used (Bragin et al., 1995). The probe $(80 \mu \mathrm{m}$ wide at the base, narrowing to $15 \mu \mathrm{m}$ at the tip) had 16 recording sites that were $100 \mu \mathrm{m}$ from each other in the vertical direction. The silicon probe was inserted into the hippocampus with the aid of a microdrive (Bragin et al., 1995). During the experiment, evoked field potentials helped guide the positioning of the microelectrodes. Two stainless steel watch screws, driven into the bone above the cerebellum, served as indifferent and ground electrodes.

Six four-channel MOSFET input operational amplifiers, mounted in the female connector, served to eliminate cable movement artifacts (Buzsáki et al., 1989). EEG was recorded $(1 \mathrm{~Hz}$ to $5 \mathrm{kHz})$ during sleep and exploration in the rat's home cage (Bragin et al., 1995) and sampled at $10 \mathrm{kHz}$ with 12-bit precision. The data were stored on optical disks and subjected to additional low-pass filtering $(<250 \mathrm{~Hz})$ before the analysis.

Data analysis. Data analysis consisted of computations of the autospectra for each signal, and the ordinary and partial coherences for different combinations of signals, similar to earlier applications of this technique for the analysis of cortical (Gersch and Goddard, 1970; Lopes da Silva et al., 1980a,b; Tucker et al., 1986; Dumermuth and Molinari, 1991; Kaminski et al., 1995, 1997; Liberati et al., 1997; Sherman et al., 1997) and hippocampal EEG (Kocsis et al., 1994; Kocsis and Vertes, 1994; Sekihara et al., 1996; Korzeniewska et al., 1997; Sherman et al., 1997). Fast Fourier Transform was performed on all 16 voltage recordings in contiguous windows of equal length, each containing 1024 data points. For each window, the autospectra for the signals, as well as the cospectrum and the quadrature spectrum, for each pair of signals were computed.

The raw spectra were smoothed using a three point moving average and averaged over the windows. The autospectra $G_{x x}(f)$ and the squared coherence spectra $K_{x y}{ }^{2}(f)$ were calculated, as described previously (Kocsis et al., 1990, 1994; Kocsis and Vertes, 1994). The partial coherences were calculated for all possible combinations of signals using the algorithms developed by Jenkins and Watts (1968). The squared partial coherence spectrum $K_{x y: z}{ }^{2}(f)$, measuring the squared covariance at frequency $f$ between two signals $X(t)$ and $Y(t)$, was calculated as

$$
K_{x y . z}{ }^{2}(f)=1-\left[1-K_{y . x z}{ }^{2}(f)\right] /\left[1-K_{y z}{ }^{2}(f)\right]
$$

where $K_{y . x z}{ }^{2}(f)$ is the squared multiple coherence spectrum, measuring the proportion of the spectrum of signal $Y(t)$ that can be predicted from signals $X(t)$ and $Z(t)$, calculated as

$$
K_{y \cdot x z}^{2}(f)=1-\underline{\underline{G}}_{x y z}(f) /\left[G_{y y}(f) \underline{\underline{G}}_{x z}(f)\right] .
$$

$\underline{\underline{G}}_{x y z}(f)$ in this expression is the determinant of the spectral matrix of three signals $X(t), Y(t)$, and $Z(t)$, and $\underline{\underline{G}}_{\mathrm{xz}}(f)$ is the determinant of spectral matrix of signals $X(t)$ and $Z(t)$. When expanded, this form may be expressed in terms of the autospectra, cospectra, and quadrature spectra of the three signals (Jenkins and Watts, 1968, their page 488). All spectra displayed in the figures have a horizontal scale of $0-30 \mathrm{~Hz}(0-15$ $\mathrm{Hz}$ in Fig. 10). The amplitude of the autospectra is autoscaled to the largest peak in any of the EEG recordings. The coherence spectra are represented on a scale of $0-1$.

For each data segment, Fourier analysis yielded 16 autospectra and a total number of 120 pairwise coherences that were arranged in a triangular-shaped matrix (Fig. 1). The vertical axis of this matrix indicates the location of the electrodes separated by $100 \mu \mathrm{m}$ from each other, and the horizontal axis corresponds to the distance between the electrodes increasing from $100 \mu \mathrm{m}$ in the first column to $1.5 \mathrm{~mm}$ in the last column. The number of coherence functions in each column changes along the horizontal axis from 15 to 1 as we go from the closest neighbors to more and more widely separated electrode pairs. Viewing this matrix of coherence functions as a map allows the identification of different anatomical and functional "zones" corresponding to different sets of signal pairs. For example, Figure $1 B$ highlights all coherence functions of field potentials recorded in and above the CA1 pyramidal layer on one hand and all other derivations on the other. Figure $1 C$ illustrates coherence functions computed between the signals recorded near the hippocampal fissure and the other 15 locations. One can also recognize a coherent zone connecting all possible pairs of signals recorded from within the dentate hilar region, as represented by a dark triangle (caused by high coherences over the entire $0-30 \mathrm{~Hz}$ frequency range, see below) in the bottom left corner of the coherence map. Similar triangular-shaped zones are also visible for alveus-stratum (str.) oriens-pyramidal layer recordings in the top left corner of the map and a somewhat less homogeneous zone around the hippocampal fissure (middle, left).

Partial coherence functions were displayed using similar maps. Each partial coherence map represents the pairwise coherence functions after the components correlated with one of the signals were eliminated. The partial coherence maps do not include coherence functions for the signal used for partialization. Sixteen partial coherence maps were constructed for each data segment containing a total of 1680 partial coherence functions. Many of the coherent spectra, however, were redundant. For example, partialization with signals derived from any of the str. oriens recording sites gave very similar results. The relevant information was extracted from the comparison of the partial coherence maps with each other and with the ordinary coherence map. The signals that exhibited significant alterations were analyzed further in signal triplets, as described previously (Kocsis, 1994; Kocsis et al., 1994).

The goal of coherence analysis, in general, is to reveal correlated oscillations in different networks and deduce functional coupling among these networks. Highly coherent oscillations between two structures can occur because they are functionally connected or because they share a common input. Ordinary coherence function cannot differentiate between these possibilities. However, the power of coherence analysis can be increased by means of partialization, as illustrated in Figure $2 A$. The various hypothetical connections among signals $x, y$, and $z$ are all associated with a high ordinary coherence between $x$ and $y$. If the high ordinary coherence can be explained by the input from $z$ (Fig. $2 A$, \#2), coherence $x-y$ will be completely eliminated by the partialization method (i.e., $x-y / z=0)$. It will not change, however, if $x$ and $y$ are tightly coupled and $z$ does not exert a significant influence on their activity (i.e., $x-y=x-y / z$ ) (\#3). When all three networks are connected (\#1), partial coherence will fall somewhere between zero and the level of the ordinary coherence. Finally, if $z$ affects $x$ and $y$ in an asymmetric manner, the coherence between two signals may increase after partialization (\#4). In this case, partialization is equivalent to the elimination of a "noise term," which differentially affects $x$ and $y$ (Lopes da Silva et al., 1980a). As the number of signals increases, the pattern of coupling between them may become more complex. However, "triangulations" among multiple signal triplets can significantly expand the power of the coherence method.

Another important feature of partialization is its ability to separate different frequency components of the signal. Coherence between two signals may increase spuriously because of the fact that both electrodes record volume-conducted signals from other sources. Because the mag- 

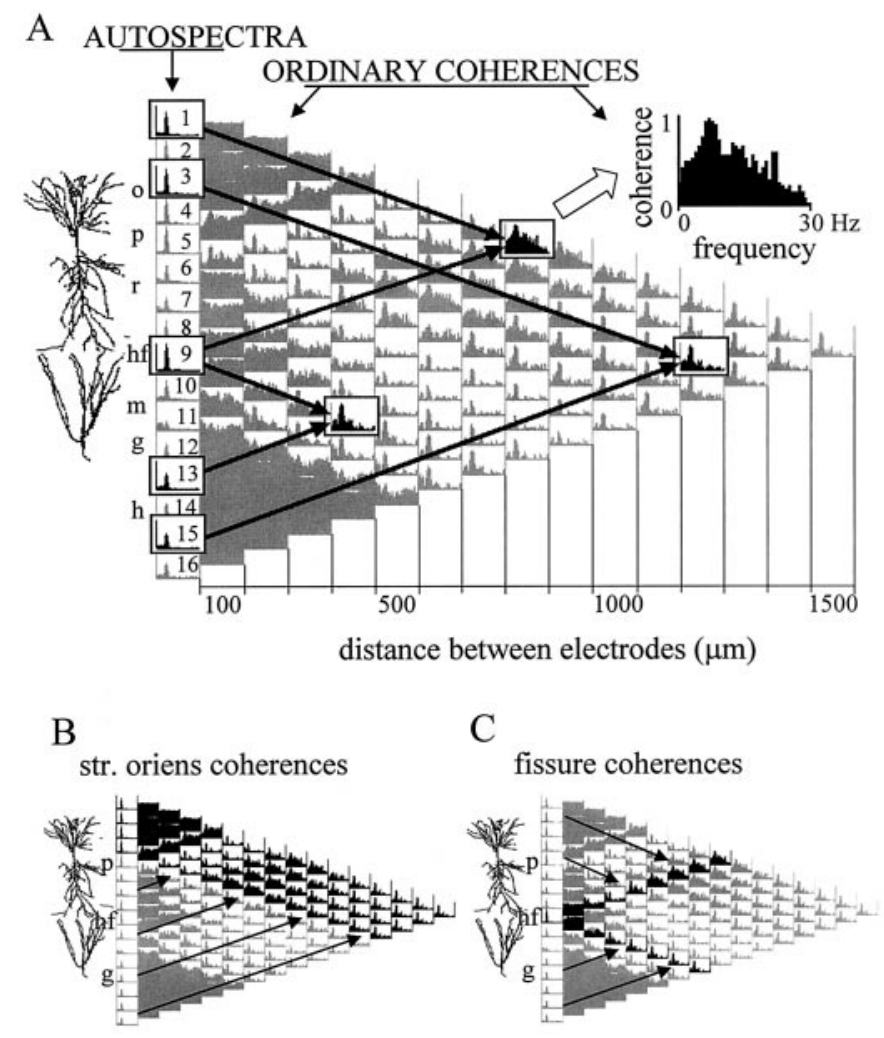

Figure 1. Ordinary coherence map of EEG activity and overview of data presentation. $A$, The 16 recording sites were equally spaced along a line between the CA1 and dentate gyrus regions. The different layers $(o$, str. oriens; $p$, pyramidal layer; $r$, str. radiatum; $h f$, hippocampal fissure; $m$, dentate gyrus molecular layer; $g$, granule cell layer; $h$, hilus) and the relative position of the principal cells is indicated left of the coherence map. The left column of the matrix shows the distribution of power (autospectrum) between 0 and $30 \mathrm{~Hz}$ for each signal, scaled to the channel with the highest power peak in the theta band (usually at the hippocampal fissure). The 120 pairwise coherence functions were arranged in a triangular matrix so that their position on the map indicates the location of the sites compared. Individual coherence functions for any signal pair are found at the point of crossing of arrows departing from the boxes containing the autospectra of these signals (in $A$, three examples are highlighted on the map). The first column of coherence functions (right of the autospectra) contains 15 traces representing the relationship between neighboring signals (100 $\mu \mathrm{m}$ spacing); the second column (14 spectra) compares second neighbors (i.e., electrode tips separated by $200 \mu \mathrm{m}$ ), etc. The coherence spectrum at the peak of the triangle map connects electrodes 1 and 16 (CA1 str. oriens-alveus and dentate hilar region, respectively; $1.5 \mathrm{~mm}$ distance). Each coherence function spans from 0 to $30 \mathrm{~Hz}$ and is scaled from 0 to 1 (insert at top). $B, C$, Two different zones of the coherence map representing all coherence functions related to field potentials recorded at any location above the CA1 pyramidal layer (highlighted in black in $B$ ) and those related to the signal recorded from the hippocampal fissure (highlighted in black in $C$ ).

nitude of coherence does not depend on the magnitude of the signals recorded, relatively weak noise, common to both electrodes, can produce a significant coherence between the recorded signals. This common noise may obscure true coherence that emerge from neuronal connections. A characteristic feature of such spurious coherence is that it is equally high (close to unity) for all frequencies. The first example in Figure $2 B$ demonstrates that partialization may selectively eliminate the volumeconducted noise component from the signals and reveal the true pattern of coherent oscillations. In this example, the dominant frequency component at all three locations was caused by theta activity (see autospectra of $x, y$, and $z$ ). Nevertheless, the coherence at theta frequency was obscured by other frequencies in the pairwise coherence functions. If the oscillatory component is only coherent between two of the three signals (in this case between $x$ and $z$ ), whereas the noise is common for all three,
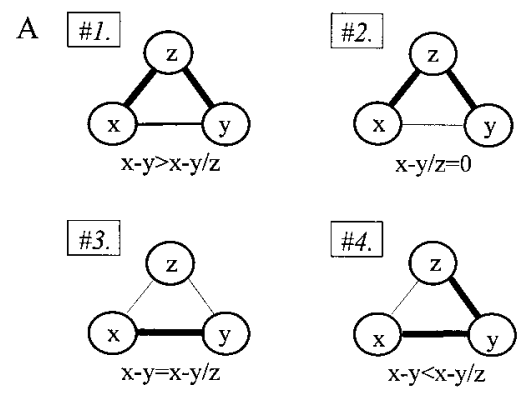

B

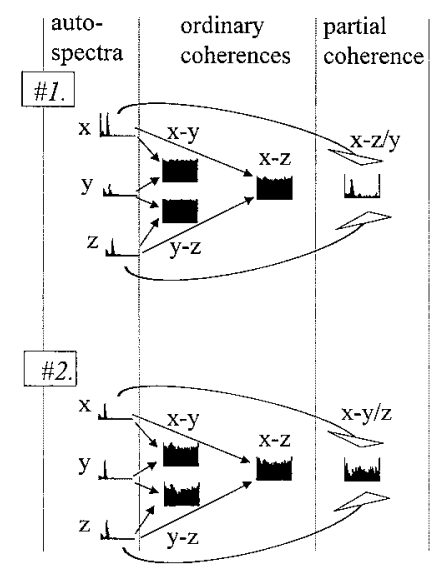

Figure 2. Features of the partial coherence analysis. A, Various combinations of anatomical connections can be revealed by the partialization technique. Different patterns of coupling (\#1-\#4) between three signals $(x, y$, and $z)$. Partialization with $z(x-y / z)$ may either decrease $(\# 1, \# 2)$ or increase (\#4) $x-y$ coherence or leave unchanged (\#3) the ordinary coherence between $x$ and $y$, depending on the relationships between the three signals. $B$, Separation of an oscillatory component (theta) between two closely placed electrodes $(x$ and $z$ ) from the wide-band noise using two different auxiliary signals. Signal $y$ in $B, \# 1$ shares only the wide-band component with $x$ and $z$. In $B, \# 2$, only the rhythmic component is common. Accordingly, partialization selectively eliminates the noise $(B$, $\# 1)$ or theta pattern coherence $(B, \# 2)$.

then partialization of $x-z$ coherence with the third signal $(y)$ can eliminate the noise and uncover a distinct peak in the residual coherence function. In the second example (Fig. 2B, \#2), the composition of the three signals was different, and partialization selectively eliminated the theta component from the homogeneous, wide-band coherence function. Note that the original matrix of pairwise ordinary coherences was very similar in both cases (Fig. 2B, \#1, \#2). Partialization, however, revealed new information and indicated the pattern of coupling among the three signals.

Histological procedures. After completion of the experiments, the rats were deeply anesthetized and perfused through the heart first with cacodylate-buffered saline, $\mathrm{pH} 7.5$, followed by a cacodylate-buffered fixative containing $4 \%$ paraformaldehyde and $5.9 \%$ calcium chloride, $\mathrm{pH}$ 7.5. Brains were left in situ for $24 \mathrm{hr}$, removed, and then post-fixed in the same solution for 1 week. The brains were sectioned with the probes left in the brain on a vibratome at $100 \mu \mathrm{m}$ in the coronal plane. The sections were stained with the Nissl method.

\section{RESULTS}

The hippocampal EEG of unanesthetized rats was simultaneously recorded at 16 vertically spaced locations in the CA1 and dentate gyrus regions (intertip distance of $100 \mu \mathrm{m}$ ) (Bragin et al., 1995). For detailed analysis, we selected four animals with the recording probes placed at various mediolateral planes in the CA1-dentate gyrus axis (Fig. $3 A$ ). The probe in rat 29 was most lateral and 

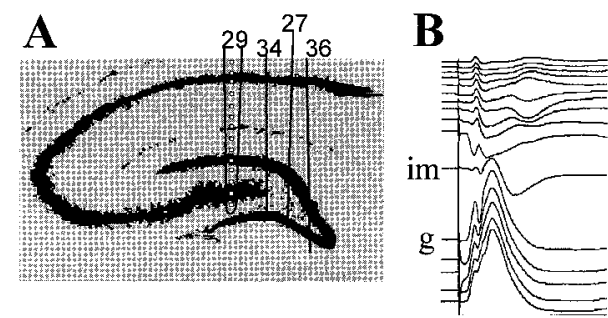

C

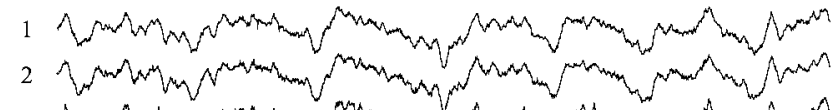

of 3 A

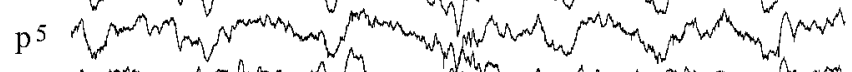

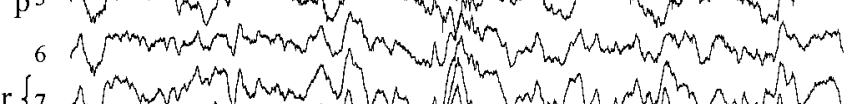

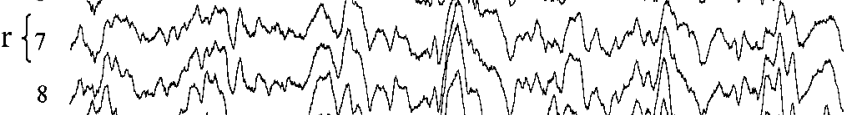

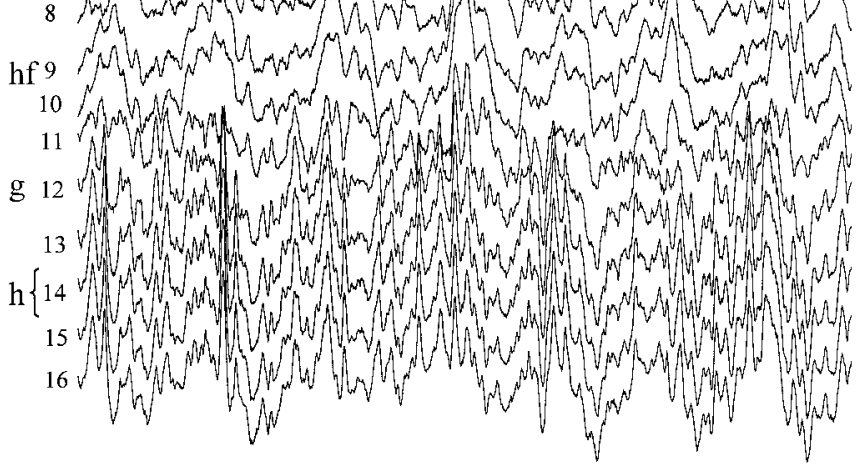

Figure 3. Simultaneous recording of evoked and spontaneous field activity in the CA1-dentate gyrus axis. $A$, Positions of the silicon probes in the rats included in this study. The traces shown in $B$ and $C$ were recorded with probe 29 . Spacing of recording sites, $100 \mu \mathrm{m}$. B, Evoked field potentials in response to commissural (right) and perforant path (left) stimulation. These evoked potential profiles were used to determine the vertical location of the recording sites. $C$, Theta activity recorded during REM sleep. $o$, Str. oriens; $p$, pyramidal layer; $r$, str. radiatum; $h f$, hippocampal fissure; im, inner third of the molecular layer; $g$, granule cell layer; $h i$, hilus.

traversed the CA1, dentate gyrus, and the CA3c regions. In rat 34, the probe passed medial to the hilar tip of the CA3c pyramidal layer, whereas in the remaining two animals (rats 36 and 27), the probes traversed the apex of the hilus and the apex of the granule cell layer, respectively. The mediolateral position of the recording probes was verified by histological processing of the brains. The exact positions of the recording sites were determined during in vivo recordings of the evoked potentials in response to perforant path and commissural input stimulations (Fig. 3B) (Bragin et al., 1995). The CA1 pyramidal layer was recognized by the presence of multiple-unit activity, large-amplitude field "ripples" during immobility and slow wave sleep (SWS) (Buzsáki et al., 1992), and evoked population spikes in response to commissural stimulation. The maximum negative field response below the pyramidal layer identified the middle of str. radiatum. The inner molecular layer of the dentate gyrus was identified by the polarity reversal of the perforant path response. The position of the hippocampal fissure was extrapolated from the electrode positions in the inner molec-

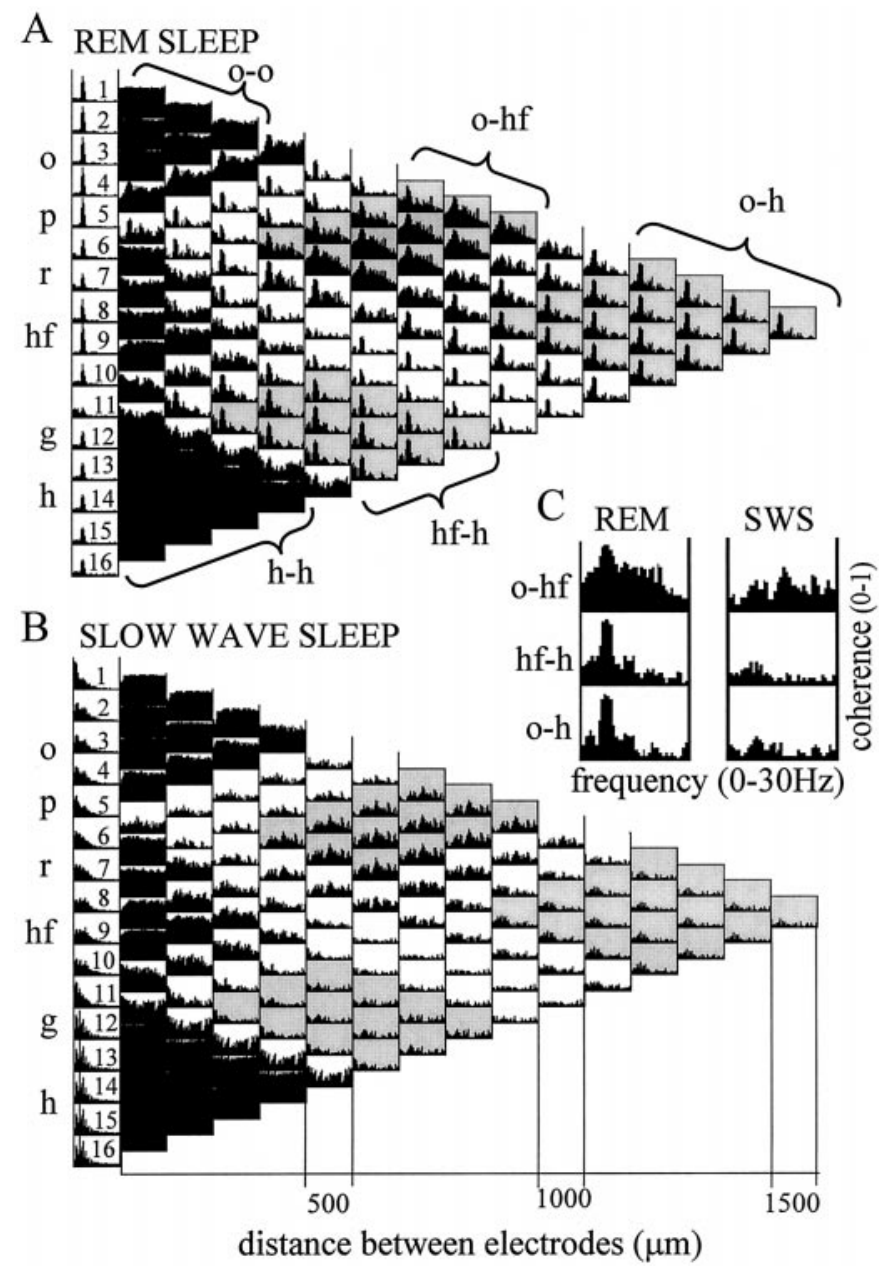

Figure 4. Autospectra (1-16) and maps of pairwise ordinary coherence functions computed from two $50 \mathrm{sec}$ segments recorded during REM sleep $(A)$ and SWS $(B)$. Coherence spectra related to three major anatomical regions are highlighted: alveus-CA1 str. oriens (signals 1-4), distal dendritic regions of CA1 pyramidal cells and dentate granule cells, straddling the hippocampal fissure $(8-10)$, and the dentate hilar region (12-16). Note similarity of the coherence functions within the respective zones. Note also that coherence functions of signals recorded within the same anatomical region showed high values over the entire $0-30 \mathrm{~Hz}$ frequency band (compare the three black triangles on the left of both maps). The three zones were separated by coherence spectra with relatively low values at all frequencies, corresponding to CA1 str. radiatum (6, 7 ) and dentate inner molecular layer (11) recordings. $C$, Pairwise coherence functions between three sample signals, recorded in different layers $(o-h f, h f-h, o-h)$, for frequencies between 0 and $30 \mathrm{~Hz}$ during REM and SWS. During REM sleep, all coherence spectra were dominated by a large peak at theta frequency. Abbreviations as in Figure 1.

ular layer and the CA1 pyramidal layer with the help of the histological sections.

\section{Ordinary coherence functions}

Pairwise coherence functions between all recording sites (ordinary coherence maps) recorded during 50-sec-long theta and nontheta segments are shown in Figure 4, $A$ and $B$, respectively. In agreement with previous observations, the largest coherence values were found between neighboring recordings (Bullock et al., 1990; Bragin et al., 1995). On both maps, three anatomically distinct zones were outlined, within which the coherence was high for any neighboring pairs of signals over the entire frequency range examined (i.e., $0-30 \mathrm{~Hz}$ ). The three anatomically segre- 
gated zones were similar in all four rats. Within alveus-CA1 strata oriens-pyramidale and within the hilus, the pairwise coherence values were equally high at 100, 200, or even $400 \mu \mathrm{m}$ tip separation [Fig. 4, channels 12-16 (ch12-ch16)]. The third zone between signals, recorded from just below the CA1 pyramidal layer and the granule cell layer of the dentate gyrus, appeared less homogeneous. Signals recorded relatively further away from each other $(\geq 300 \mu \mathrm{m})$ were less coherent than nearer neighbors. The distance between different recording sites, however, was not the major factor determining the strength of relationship between the EEG signals. The coherence functions between CA1 str. radiatum and other recordings and dentate inner molecular layer and other recordings were generally low, resulting in a remarkable segmentation of the coherence map. For example, coherence values between recording site $2(\operatorname{ch} 2)$ and other sites decreased from str. oriens to the proximal radiatum but increased again toward the hippocampal fissure.

Ordinary coherence functions that characterize the relationship between signals recorded in different zones were limited to certain frequency bands. During REM sleep, high ordinary coherences were found between most signal pairs at theta frequency (Fig. 4A). This pattern was represented by relatively narrow peaks at the dominant frequency and its harmonics. The value of theta coherence was $0.8-0.9$ for the majority of signal pairs. However, relatively low (0.5-0.6) theta coherence was found between any recordings paired with signals derived from either the dentate inner molecular layer or CA1 str. radiatum. The lowest coherence values at theta frequency $(0.2-0.3)$ were obtained between signals derived from the dentate inner molecular layer and CA1 str. radiatum.

The strongest coupling occurred between signals in the str. oriens (ch1-ch4) and the recordings at or near the hippocampal fissure (ch8-ch10). In addition to the theta peak, these signal pairs exhibited relatively high coherence values over a wider frequency range (between 0 and $30 \mathrm{~Hz}$ ) in both REM and SWS. Lower but significant nontheta coherence values were also found at low frequencies (i.e., below $10 \mathrm{~Hz}$ ) between signal pairs connecting the hilus (ch13-ch16) and granule cell layer (ch12) to recordings in the str. oriens and at the hippocampal fissure.

\section{Coherent signals between stratum oriens and hippocampal fissure}

Similar to previous observations, theta waves were of largest amplitude in the vicinity of the hippocampal fissure. The laminar profile of theta power also showed a second, smaller amplitude peak in the CA1 pyramidal layer (Buzsáki et al., 1986; Brankack et al., 1993; Ylinen et al., 1995). Coherence functions indicated a distinctly strong coupling between these two regions (i.e., relatively wide-band significant coherence during both REM and SWS).

Figure $5 A$ compares pairwise coherence functions for a signal triplet recorded from the CA1 str. oriens, hippocampal fissure, and hilus. Coherence peaks at theta frequency among these regions were not significantly different in the ordinary coherence comparisons but were nonuniformly affected by partialization. There was only a small decrease in coherence between signals recorded from str. oriens and hippocampal fissure (Fig. 5A, left), whereas theta coherence between hilus and either of the other two recording sites were completely eliminated (Fig. 5A, right). Thus, the coherence between signals recorded from the hilus and the other two sites could be predicted from the variance carried by the third recording site. This observation indicates that the
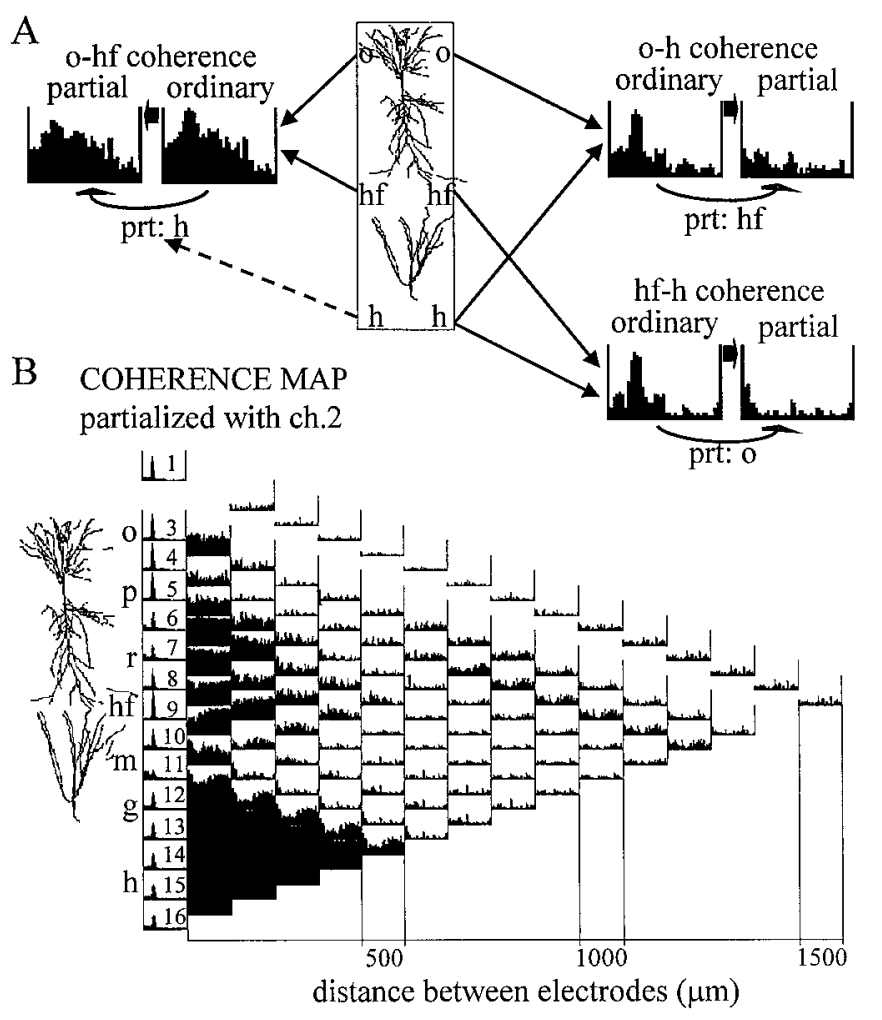

Figure 5. Coupling between theta field oscillations in str. oriens $(o)$ and hippocampal fissure $(h f)$. $A$, The pattern of relationships between recordings from str. oriens, hippocampal fissure, and hilus $(h)$ during REM sleep was tested by comparing theta coherences of each possible signal pairs before (ordinary) and after ( partial) partialization with the third signal. High ordinary coherence was found at theta frequency for all three signal pairs, which could be eliminated by partialization with either hippocampal fissure (prt: $h f)$ or str. oriens ( prt: o) signals. High theta coherence remained between str. oriens and hippocampal fissure recordings, however, after partialization with hilus signal $(p r t: h)$. B, Partial coherence map showing pairwise residual coherence after elimination of the components, which were coherent with field potentials recorded in str. oriens (signal 2). Compare the partial coherence map with the ordinary coherence map computed from the same data (Fig. $3 A$ ).

signals in str. oriens and hippocampal fissure represent a single common theta oscillator (cf. Kocsis, 1994).

Similar results were found for other signal triplets as well, in which the hilus recording was replaced with signals recorded from any other site, except the str. oriens and hippocampal fissure locations. In general, theta coherence between oriens and hippocampal fissure (Fig. 5, ch9) recordings was not affected by partialization with signals outside these locations. On the other hand, theta coherence values decreased significantly when signals recorded in the str. oriens (ch1-ch4) or at the hippocampal fissure (ch9) were used for partialization. Partialization with these signals completely eliminated the theta component from all coherence functions (for exceptions, see below). The strong effect of partialization, using a str. oriens recording site, is demonstrated in Figure $5 B$. After partialization, significant coherence remained only between neighboring signals recorded within the same zones (see dark triangles around the hippocampal fissure and in the hilus).

For a more refined localization of the potential theta inputs, EEG recorded from the str. oriens and around the hippocampal fissure was further examined in signal triplets taken from these zones. The ordinary coherence functions (Figs. 4A, 6A, top) 

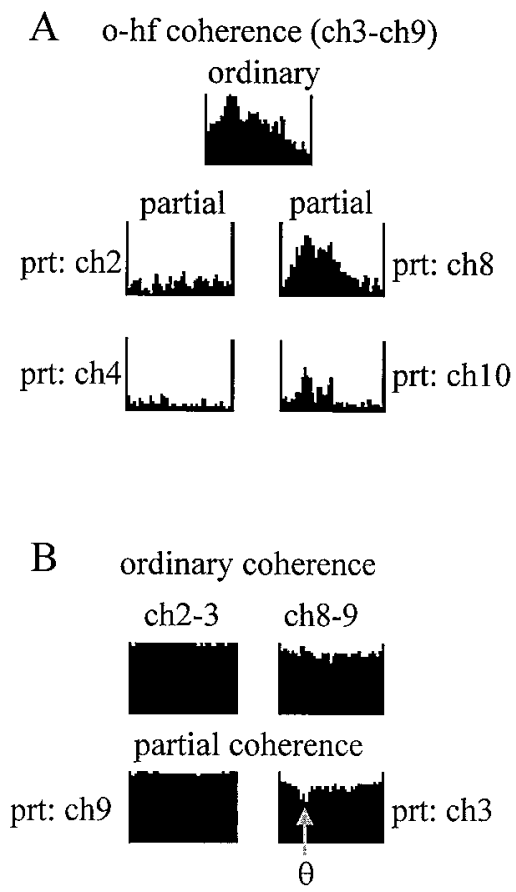

frequency: $0-30 \mathrm{~Hz}$

Figure 6. Spatial resolution of the partialization technique. A, Effect of partialization on the str. oriens-hippocampal fissure coherence using neighboring signals recorded at $100 \mu \mathrm{m}$ distance on either side of the str. oriens and hippocampal fissure electrodes. High theta peak present in the ordinary coherence function between hippocampal fissure (ch9) and any one of the str. oriens sites (ch3, top) was eliminated by partialization with signals recorded from within str. oriens (ch2 and ch4, left) but not with signals recorded in distal str. radiatum $(\operatorname{ch} 8)$ or dentate molecular layer $($ ch10, right). B, The high coherence, homogeneous in the entire $0-30 \mathrm{~Hz}$ range, between neighboring signals in the str. oriens were not affected by partialization with signals recorded from outside str. oriens, including that in hippocampal fissure $(\operatorname{ch} 9$, left $)$. On the other hand, theta oscillations in the str. oriens explained some of the coherence between hippocampal fissure and its closest neighbors because partialization with ch3 (o) specifically decreased the coherence between ch9 (hippocampal fissure) and ch8 (str. lacunosum moleculare) at theta frequency.

between any recordings from the str. oriens (ch1-ch4) and the hippocampal fissure area (ch8-ch10) were nearly identical, consisting of a $0-30 \mathrm{~Hz}$ wide-band component and a large, sharp theta peak $(0.8-0.9)$. The coherence between ch9 and oriens signals could not be eliminated by partialization with signals derived from nearby locations (i.e., the dentate molecular layer, ch10; the distal str. radiatum, ch8). On the other hand, partialization with a str. oriens signal completely eliminated all coherences from other str. oriens-hippocampal fissure signal pairs (i.e., ch2-4 and ch9; Fig. 6A, left). Furthermore, the high ordinary coherence among str. oriens recordings was not changed by partialization with ch9 (Fig. 6B, left). Finally, the theta signal recorded from the hippocampal fissure site (ch9) was more coherent with EEG recorded from str. oriens than from its closest neighbors, as revealed by the decreased coherence at theta frequency after partialization with the signal recorded from ch3 (Fig. 6B, right). This analysis revealed that the source of theta coherence in the hippocampal fissure region could be precisely localized to one electrode (ch9). In contrast, the effect of partialization on the magnitude of theta coherence was similar, with all recording sites within the str. oriens. In summary, theta activity recorded in the str. oriens and at the hippocampal fissure explained most of the variance in all other layers of the hippocampus. ordinary coherence

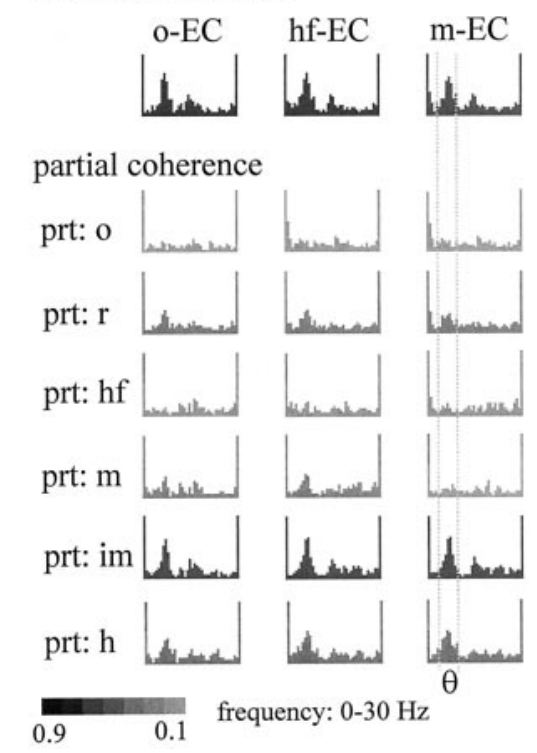

Figure 7. Coherence in the theta band between a recording site in the entorhinal cortex $(E C)$ and different layers of the hippocampus. The ordinary coherence peak at theta frequency could be eliminated by partialization with signals in str. oriens ( prt: $o$ ) or hippocampal fissure ( prt: $h f$ ). Significant residual theta coherence remained, however, after partialization with signals in the str. radiatum ( prt: $r$ ), the midmolecular layer ( prt: $m$ ), the inner molecular layer ( prt: im), or the hilus ( prt: $h$ ). Note that partialization with the inner molecular signal did not affect theta coherence between entorhinal cortex and at site $100 \mu \mathrm{m}$ from the inner molecular layer $(m-E C)$.

In two experiments, EEG activity of the entorhinal cortex (layer II/III) was recorded simultaneously with hippocampal EEG. Theta activity in the entorhinal cortex showed a significant coherence $(0.3-0.5)$ with all hippocampal recordings. The highest theta coherence was found in pairs containing signals from the str. oriens, hippocampal fissure, and the dentate molecular layer. Partialization with these signals eliminated the entorhinal cortexrelated theta coherence at all recording sites. Conversely, partialization with signals outside of these three layers did not change the entorhinal cortex-hippocampal theta coherence (Fig. 7). Furthermore, analysis of the entorhinal cortex-related theta coherence in the signal triplet, recorded from CA1 str. oriens, hippocampal fissure, and dentate molecular layer, indicated that the str. oriens-hippocampal fissure theta component originated more likely from the hippocampal fissure site than from sites in the str. oriens. This is indicated by the finding that partialization with the signal from the dentate molecular layer (Fig. 7, prt: $m$ ), which also receives a direct input from the entorhinal cortex, had a stronger effect on entorhinal cortex-str. oriens coherence than on the entorhinal cortex-hippocampal fissure coherence.

\section{Coherent theta signals between str. radiatum and the inner molecular layer of the dentate gyrus}

Elimination of the entorhinal cortex-mediated theta component, which dominated the electrical activity in most layers of the hippocampus, also enabled us to uncover theta oscillations that appeared independent of the entorhinal cortex input. These theta waves were present in the CA1 str. radiatum and inner molecular layer of the dentate gyrus, i.e., the target layers of the intrahippocampal associational inputs.

Theta signals in the CA1 str. radiatum and dentate inner 
molecular layer were different from all other recordings. They showed the lowest ordinary coherence at theta frequency (Fig. $4 A$ ). Partialization with these signals exerted a negligible effect on the coherence map, except on pairs involving other str. radiatum recordings. In addition, the weak coherence between str. radiatum and dentate inner molecular layer represented the only theta component that could not be eliminated by partialization with signals derived from the hippocampal fissure or str. oriens (Fig. 5B).

In the analysis shown in Figures 8 and 9, we tested the hypothesis that the magnitude of coherence between two signals can increase after partialization, provided that the partializing signal has an asymmetric relationship to the signals to be compared (compare with Fig. 2A, \#4). Theta coherence between signals derived from str. radiatum and dentate inner molecular layer increased from 0.25 to 0.67 and 0.81 by partialization with signals recorded from other dentate molecular layer and the str. radiatum sites, respectively (Fig. $8 A$ ). Partialization with signals recorded at the same distance but from a different anatomical layer had a lesser effect (Fig. 8A, ch5 and ch12). The largest relative increase of theta coherence between str. radiatum and inner molecular recordings occurred by partialization with the signal recorded at the hippocampal fissure (Fig. $8 B$ ). This effect can be interpreted as follows. First, the signal recorded at the hippocampal fissure reflected mostly the theta component derived from the entorhinal cortex, as shown above. Second, we assume that the recording sites at the hippocampal fissure and the inner molecular layer shared a common noise. Third, we assume that the intrahippocampal associational inputs to str. radiatum and inner molecular layer were weak and did not spread to the hippocampal fissure electrode. As a result, partialization with the hippocampal fissure EEG resulted in an increase of the coherence between signals derived from the str. radiatum and the inner molecular layer.

In rat $\mathrm{BA} 36$, the electrode array passed through both the dorsal and ventral blades of the dentate gyrus (Fig. $3 A$ ). Accordingly, partialization increased the coherence between signals recorded from CA1 str. radiatum and inner molecular layer of either the dorsal or ventral leaf of the dentate gyrus (Fig. 9).

\section{Relative independence of entorhinal cortex-mediated and intrahippocampal theta oscillators}

The relative independence of theta signals recorded in the target layers of the entorhinal cortex and intrahippocampal associational afferents was also revealed by examining the voltage variation of the theta signals in the various layers. Although theta power in the str. oriens recordings was significantly less than at the hippocampal fissure, the relative power changes with time were relatively small $(<20 \%)$. Furthermore, the recordings from str. oriens reliably predicted the power changes in the hippocampal fissure signal $(r=0.84)$ (Fig. 10A). In contrast, recordings from str. radiatum showed a large power variability relative to the hippocampal fissure theta. In fact, the calculated $z$-scores of theta power $(6-8 \mathrm{~Hz})$ revealed a reciprocal relationship between the amplitude of theta recorded from these respective layers $(r=$ -0.25 ) (Fig. 10B).

\section{Effect of entorhinal cortex lesion on theta coherence in the hippocampus}

The effect of entorhinal cortex lesion on theta coherence was tested in all four rats. As reported previously (Bragin et al., 1995), theta activity was present during exploratory waking and REM sleep immediately after the lesion. However, the amplitude of theta was reduced by $50-70 \%$. In absolute values, the reduction was most prominent at the level of the hippocampal fissure and in the pyramidal layer-str. oriens. The largest power in the theta band now occurred in the CA1 str. radiatum and the hilus (Fig. $11 A$, autospectra).

Parallel with the power decrease of theta oscillation, the relative power of the wide-band noise significantly increased, resulting in relatively homogeneous and high coherence values among all signals below the CA1 pyramidal layer. These coherence functions were dominated by a wide-band component covering the entire $0-30 \mathrm{~Hz}$ range (Fig. 11A). The presence of a noiseindependent theta component could be verified only by elimination of the common wide-band noise by partialization, as shown in Figure $11 B$.

In contrast to the intact animal, EEG in the str. oriens showed high coherence with signals recorded from all layers, including the str. radiatum and the dentate inner molecular layer (Fig. $12 A$ ). In fact, theta oscillation in the str. oriens was coupled most closely with those in the str. radiatum, as revealed by partial coherence analysis of signal triplets (Fig. 12B).

Coherent theta oscillations recorded in the layers receiving intrahippocampal associational inputs, i.e., the CA1 str. radiatum and dentate inner molecular layer, remained unaltered after removal of the entorhinal cortex. This was not obvious from the ordinary coherence functions, because the two layers appeared within the same homogeneous zone dominated by wide-band coherence. However, coherent coupling between these layers was clearly indicated by the partial coherence analysis. Figure $13 \mathrm{~A}$ illustrates the effect of partialization with different signals on the coherence between dentate inner molecular layer and its neighbors. Although the ordinary coherence values were very similar among these signals, theta coherence between the inner molecular layer and its neighbors could only be eliminated by partialization with signals recorded in the CA1 str. radiatum. Partialization with str. oriens signals had no effect, and partialization with hilar and other molecular layer recordings mostly reduced the coherence of the wide-band component (Fig. 13A). On the other hand, theta coherence was completely eliminated from signals derived from the inner molecular layer and hippocampal fissure by partialization with a signal recorded from an electrode halfway between them (Fig. 13A, prt: $m$ ). The largest effect of partialization on signal pairs involving the inner molecular layer was obtained by using a the signal from the CA1 str. radiatum (Fig. $13 B)$. Theta coherence was often reduced to almost zero.

\section{DISCUSSION}

The present experiments revealed highly coherent coupling between theta signals recorded (1) from the hippocampal fissure and str. oriens of the CA1 region and (2) between CA1 str. radiatum and the dentate molecular layer. The results of partial coherence analysis indicated that rhythmic input from the entorhinal cortex explained theta coherence between signals recorded from the hippocampal fissure and str. oriens but not the coherence between signals derived from str. radiatum and the dentate molecular layer. After bilateral lesion of the entorhinal cortex, all signals recorded from both below and above the CA1 hippocampal pyramidal cell layer became highly coherent. These observations suggest the presence of two, relatively independent, theta generators in the hippocampus, mediated by the entorhinal cortex and the intrahippocampal association circuitry, respectively. 
A

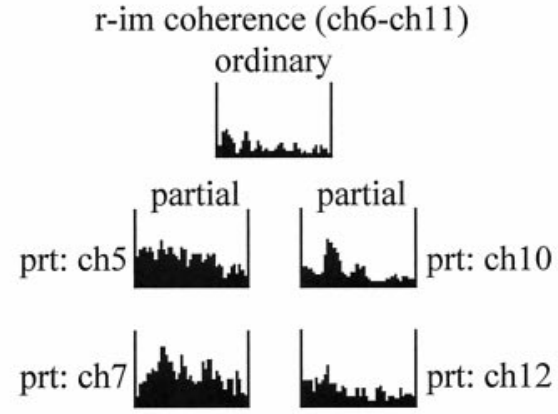

B

Partialization with hf

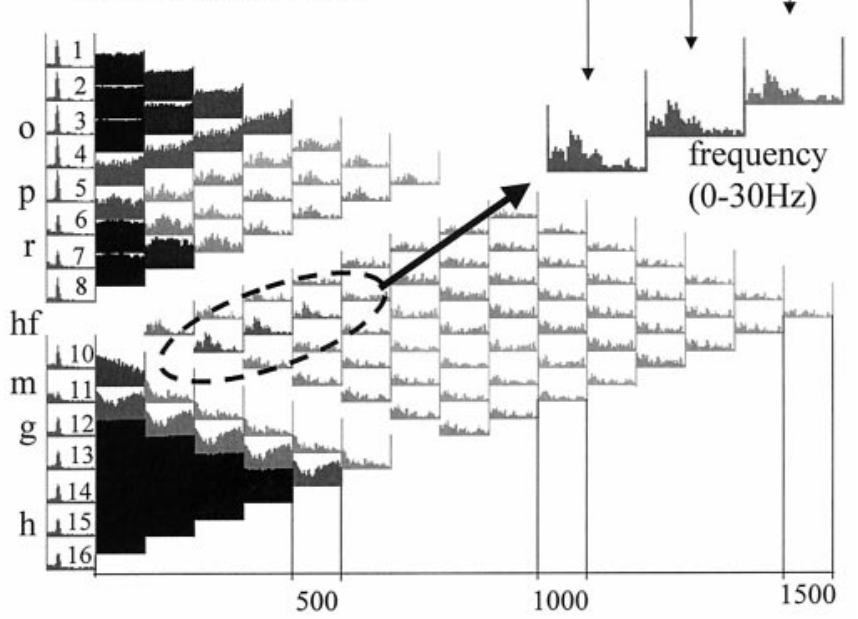

theta coherence

distance between electrodes $(\mu \mathrm{m})$
0.1

Figure 8. Coherence between signals recorded from CA1 str. radiatum $(r)$ and dentate inner molecular ( $\mathrm{im}$ ) layers. $A$, Effect of partialization on the str. radium-inner molecular ordinary coherence using neighboring signals recorded $100 \mu \mathrm{m}$ on either side of radiatum (left) or inner molecular (right) electrodes. Ordinary coherence between ch6 and ch11 was relatively low in the entire $0-30 \mathrm{~Hz}$ frequency range (top), including the theta band. Elimination of the locally shared components (with ch5 and ch7) from the $r$ (ch6) recording increased radiatum-inner molecular pairwise theta coherence (see Results for more details). Similar enhancement of theta coherence was obtained after elimination of the local components with its neighbors (ch10 and ch12) from the inner molecular (ch11) recording. The largest increase in theta coherence occurred after partialization with ch7 or ch10. $B$, Coherence map after partialization with hippocampal fissure signal (ch 9). Grayscale indicates the magnitude of the coherence value at the theta frequency. The lowest ordinary coherence values (inset) were found between the inner molecular and the recordings from different locations in radiatum ( $p r$, proximal radiatum; $d r$, distal radiatum; $l m$, str. lacunosum moleculare). These ordinary coherence functions did not have peaks at theta, whereas all the others exhibited strong theta coherence (Fig. $3 A$ ), which could be eliminated by partialization with the hippocampal fissure signal. On the other hand, partialization with signals close to inner molecular (including ch9 as shown here) eliminated a substantial part of the inner molecular signal (see relatively high ch9-ch11 ordinary coherence in the entire $0-30 \mathrm{~Hz}$ range in Fig. $3 A$ ), thereby uncovering a weak theta component unrelated to the hippocampal fissure signal. This "uncovered" theta was specifically localized to inner molecular and was coherent only with the radiatum recordings.

\section{ORDINARY COHERENCE}

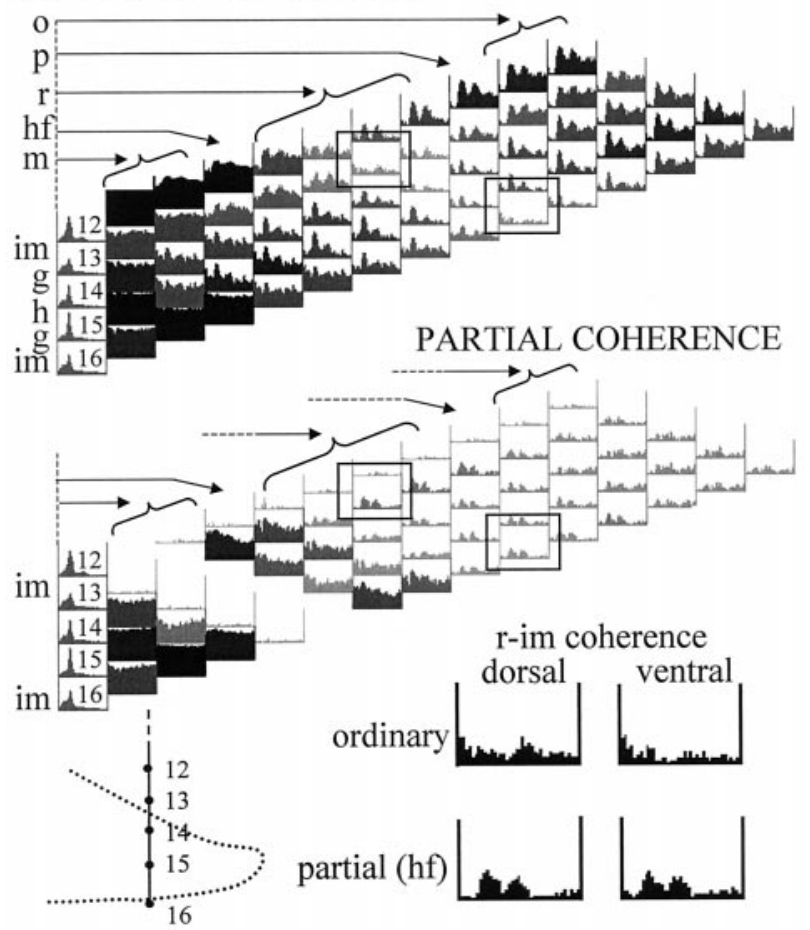

Figure 9. Coherent theta oscillation between CA1 str. radiatum $(r)$ and dentate inner molecular $(\mathrm{im})$ revealed by partialization with hippocampal fissure signal (i.e., after eliminating the entorhinal cortex-mediated theta) in a rat with the silicon probe placed medially (BA36 in Fig. 1). Grayscale indicates the magnitude of the coherence value at the theta frequency. Coherence between signals recorded from str. radiatum $(r)$ and inner molecular layer $(\mathrm{im})$ of both the dorsal and ventral blades of the dentate gyrus showed increased coherence at theta frequency after partialization with the hippocampal fissure signal.

\section{Theta signals from hippocampal fissure and CA1 stratum oriens are tightly coupled}

Previous recordings from the hippocampus of behaving rats have revealed that signals recorded in and above the CA1 pyramidal layer are identical in phase and are highly coherent. The power of the theta signal gradually increases and reaches a maximum in the pyramidal cell layer. The phase of theta begins to shift just below the pyramidal layer, coinciding with a minimum of both theta power and coherence. Deeper to this zone, the power increases again and reaches the largest maximum at the level of the hippocampal fissure, accompanied by a full reversal of the theta cycle and increased coherence at theta frequency (Winson, 1974; Bland et al., 1975; Buzsáki et al., 1983, 1986; Brankack et al., 1993; Ylinen et al., 1995). The coherent theta signal in the alveus-str. oriens and pyramidale is believed to reflect a perisomatic dipole formed by inhibitory interneurons innervating the somata of pyramidal cells (Fujita and Sato, 1964; Artemenko, 1972; Leung and Yim, 1986; Fox, 1989; Soltesz and Deschenes, 1993; Cobb et al., 1995; Ylinen et al., 1995; Toth et al., 1997; Kamondi et al., 1998b; but see Nunez et al., 1987, 1990; Konopacki et al., 1992). In contrast, the large amplitude theta signal near the hippocampal fissure is thought to reflect rhythmic dendritic depolarization of CA1 pyramidal cells (active sink) by the perforant path input, because the neurons providing afferents to str. lacunosum moleculare, i.e., layer III pyramidal cells of the entorhinal cortex, discharge phase-locked to the theta rhythm 

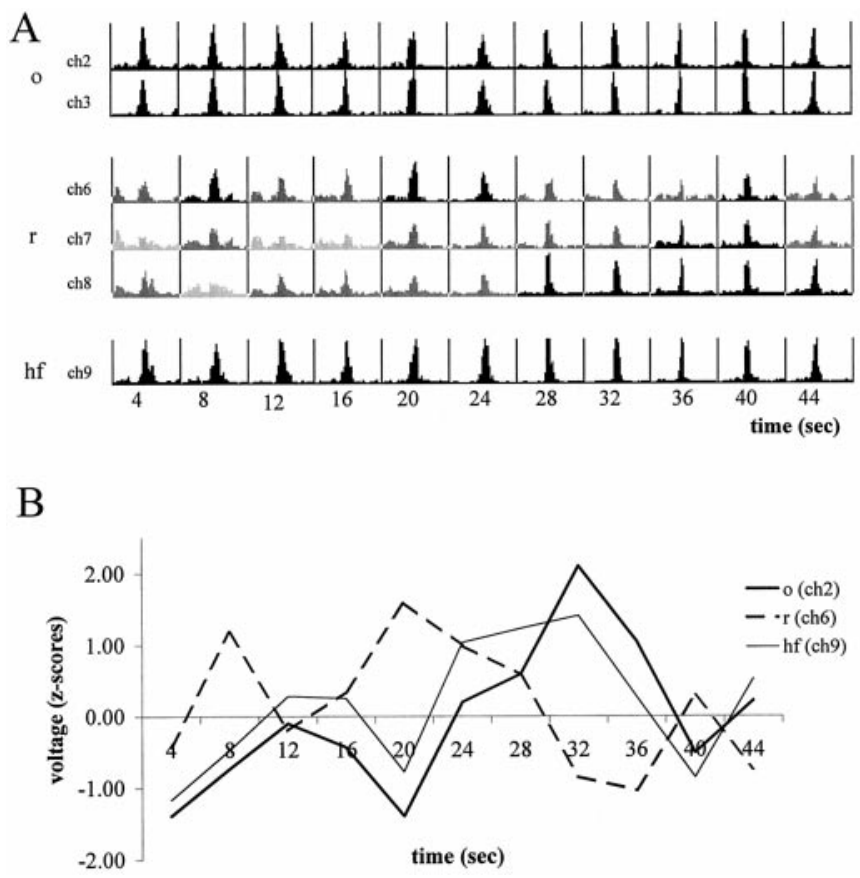

Figure 10. Relative independence of theta activity in different layers. $A$, Autospectra of theta activity during successive 4 sec epochs. In each row, the values are expressed as a percentage of maximum theta power (integrated $6-8 \mathrm{~Hz}$ ). Note similar variability of theta power in str. oriens $(o)$ and hippocampal fissure $(h f)$ recordings and different variability in the str. radiatum $(r) . B, z$-Scores of theta voltage (root mean square) in the different layers. Note reciprocal relationship between hippocampal fissure and str. radiatum.

(Mitchell et al., 1982; Alonso and Garcia-Austt, 1987; Chrobak and Buzsáki, 1998).

It is not understood, however, how the somatic inhibitory and distal dendritic excitatory theta dipoles are coordinated to produce highly coherent signals. Previous models suggested that a septal pacemaker input is responsible for the synchronous theta oscillation in both hippocampus and entorhinal cortex. According to the model, interneurons, innervating the somata of CA1 pyramidal neurons, are paced by their direct septal input (Buzsáki et al., 1983; Leung, 1984; Bland, 1986; Buzsáki et al., 1986; Alonso and Garcia-Austt, 1987; Lopes da Silva et al., 1990; Stewart and Fox, 1990; Brankack et al., 1993; Lee et al., 1994; Ylinen et al., 1995). It has been tacitly assumed that the coordinated discharge of cholinergic and GABAergic neurons in the medial septum and the diagonal band of Broca impose their coordinated pacemaker output on the principal cells and interneurons in their target structures (Buzsáki et al., 1983; Freund and Antal, 1988; Stewart and Fox, 1990; Toth et al., 1997). A caveat of this model is the lack of firm experimental support for the coordinated activity of septal neurons. Indeed, work on anesthetized and awake animals reported that septal neurons can discharge at virtually any phase of the theta cycle, and their summed activity does not necessarily provide a coherent rhythm (Gogolak et al., 1968; Apostol and Creutzfeldt, 1974; Gaztelu and Buno, 1982; Stewart and Fox, 1989; Barrenechea et al., 1995; Brazhnik and Fox, 1997; King et al., 1998).

Recent experiments indicate that the peak of dendritic depolarization during theta coincides with maximum inhibition at the soma of pyramidal cells (Kamondi et al., 1998b). In the freely moving rat, pyramidal cells and interneurons were found to
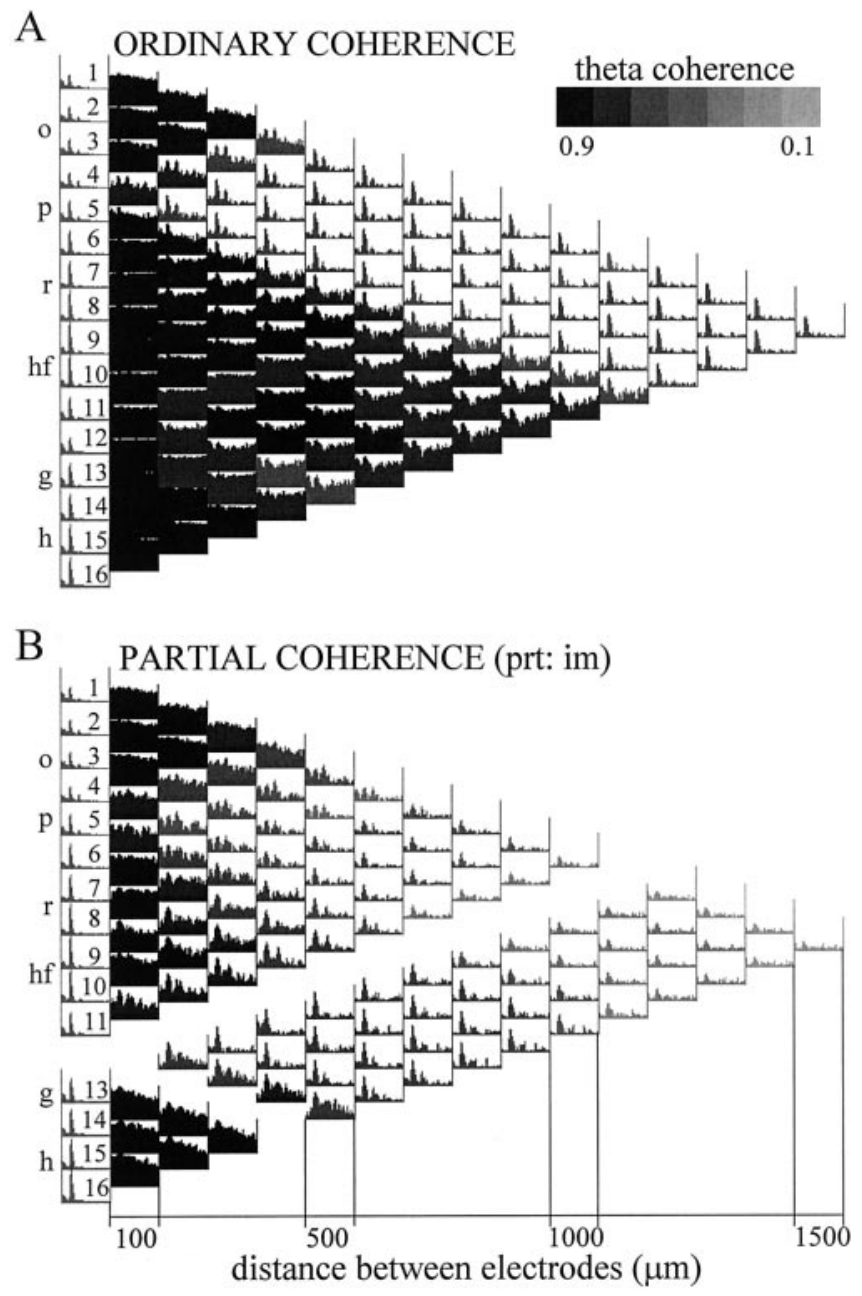

Figure 11. Effect of entorhinal cortex lesion on the coherence map of intrahippocampal signals. $A$, Ordinary coherence map of pairwise coherence functions in an animal after entorhinal cortex lesion (same rat as shown in Figs. 3 and 4). The largest power theta peaks were recorded in the str. radiatum and the hilus. High coherence was found between all signals recorded below the CA1 pyramidal layer, in the entire $0-30 \mathrm{~Hz}$ frequency band. As a consequence, clear theta peaks could be identified only in coherence functions comparing signals within this apparently homogeneous zone (5-16) with signals outside of this zone (1-4), i.e., above and below the CA1 pyramidal layer. $B$, Theta coherence buried in the homogeneous ordinary coherence functions could be revealed, however, by selectively eliminating the wide-band noise using the partialization technique. Partialization with the signal recorded from the inner molecular layer of dentate gyrus ( prt: im), for example, uncovered strong theta coherence between the hilar region and the different layers of the CA1-dentate fields. Partialization with inner molecular also decreased the theta peak between str. oriens versus other recordings.

discharge on the same phase of the theta cycle (Buzsáki et al., 1983; Fox et al., 1986; Skaggs et al., 1996; Csicsvari et al., 1999). In principle, the distal dendritic and somatic coherence of the theta signal can be explained by the common pacemaker activity of the septum in both hippocampus and entorhinal cortex. However, we also found that highly coherent coupling between EEG signals derived from electrodes near the hippocampal fissure and str. oriens was true not only for theta oscillation but for slow and faster EEG patterns as well, including the gamma band (40-100 $\mathrm{Hz}$ ) (our unpublished observations). These patterns are not paced by the septum. Interneuron-mediated inhibition of the principal cells may also be brought about by the entorhinal input. 


\section{A}
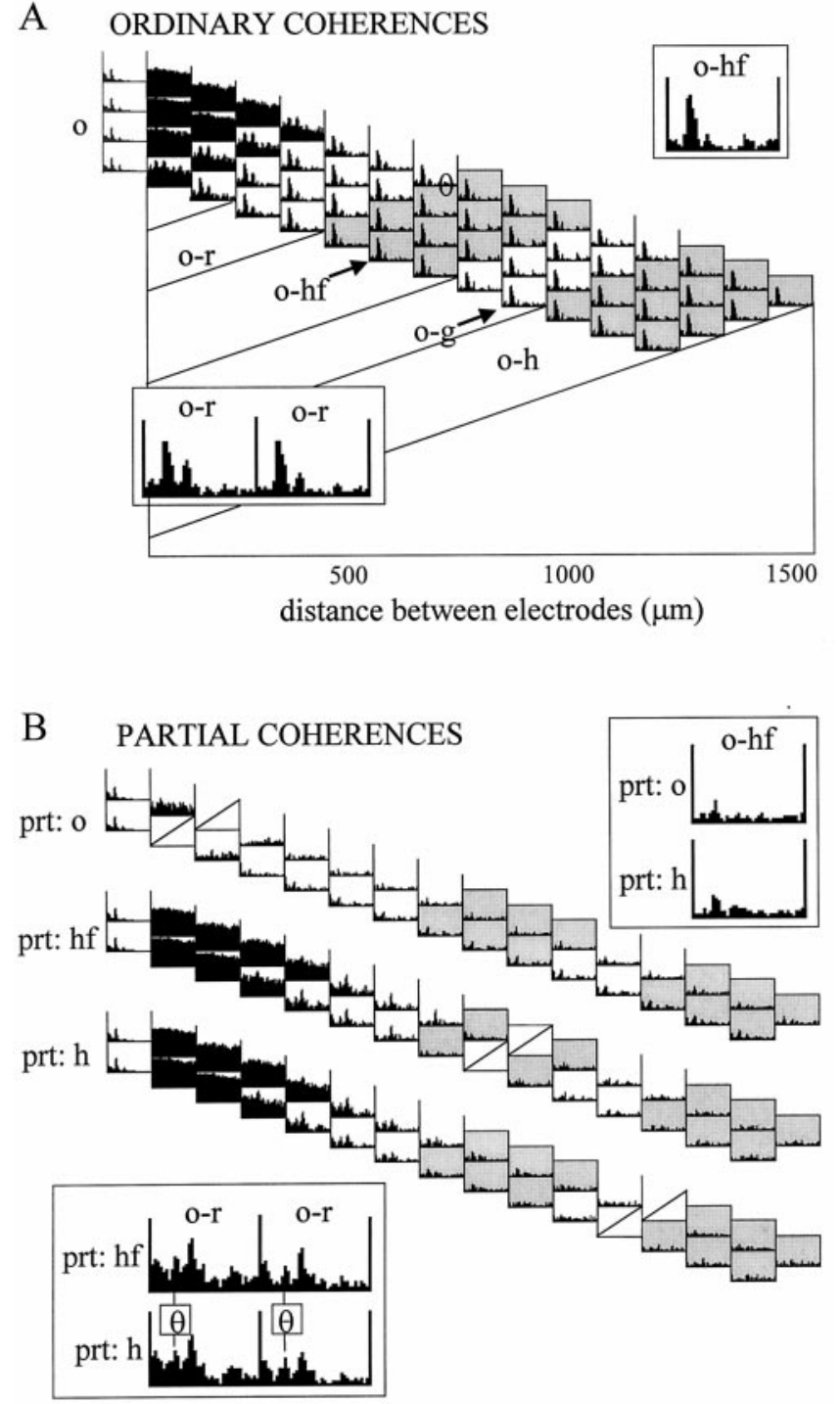

Figure 12. Coherence between theta oscillations in str. oriens $(o)$ and other hippocampal layers after entorhinal cortex lesion. $A$, The shape of all ordinary coherence functions between str. oriens $(o)$ and other recordings were similar, consisting of a relatively narrow peak at theta frequency. In contrast to the intact rat (Fig. $3 A$ ), coherence between str. oriens and hippocampal fissure was not stronger than str. oriens and radiatum coherence. $B$, As in the intact rat, theta coherence between str. oriens and other layers could be eliminated by partialization with another str. oriens signal. After entorhinal cortex lesion, however, the effect of partialization with signals outside the str. oriens was different. Partialization with hippocampal fissure and hilus signals now had a similar effect (insets).

Basket cells and chandelier cells may be particularly important in this mechanism because they possess a large apical tuft in the str. lacunosum moleculare (Li et al., 1992; Buhl et al., 1994) and can convey strong somatic inhibition to the principal cells from the entorhinal cortex (Buzsáki and Eidelberg, 1982; Colbert and Levy, 1992; Soltesz and Deschenes, 1993; Buzsáki et al., 1995; Kiss et al., 1996). This feedforward system can (1) explain the presence of distal dendritic excitatory and perisomatic inhibitory dipoles, (2) account for the strong coherent coupling between the theta signals recorded from the str. lacunosum moleculare and oriens and (3) explain the sparse discharge of pyramidal cells during theta (Fig. 14).

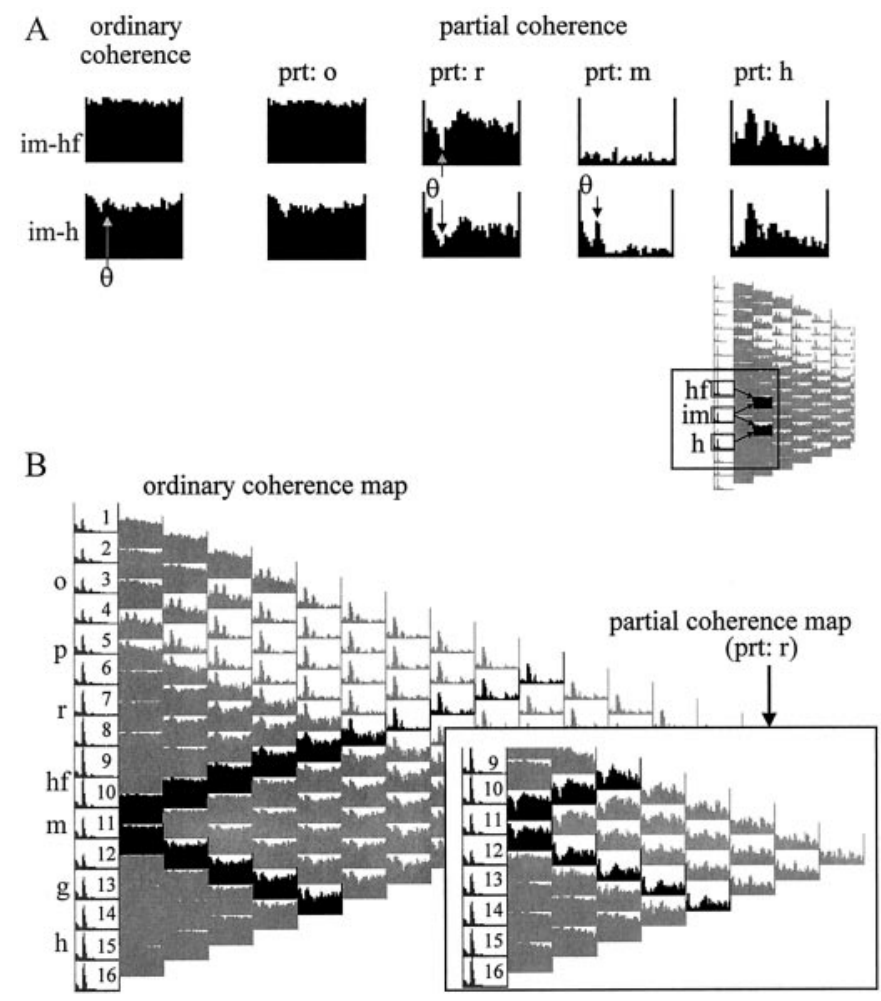

Figure 13. Coherence between theta oscillations in str. radiatum $(r)$ and dentate inner molecular layer $(\mathrm{im})$ after entorhinal cortex lesion. $A$, Changes in coherence function between inner molecular and locations $200 \mu \mathrm{m}$ above (hippocampal fissure, $h f$ ) or below (hilar region, $h$ ). Ordinary coherence values were high in the entire $0-30 \mathrm{~Hz}$ band and were not affected by partialization with signals in str. oriens (prt: $o$ ). Partialization with radiatum ( $p r t: r$ ) selectively decreased inner molecular-hippocampal fissure and inner molecular-hilus coherence at theta frequency but did not change the wide-band coherence. Inner molecularhippocampal fissure coherence values, on the other hand, were eliminated at all frequencies by partialization with a signal recorded from between the hippocampal fissure and the inner molecular layer in the dentate molecular layer (prt: $m$ ). Partialization with hilus signal (prt: $h$ ) decreased the wide-band component of the coherence function but had no effect on the theta coherence. $B$, Partialization with radiatum ( $p r t: r$, inset) selectively eliminated theta coherence between the inner molecular and all other signals.

\section{Contribution of the intrahippocampal association system to theta generation}

Activation of the recurrent excitatory network of the CA3 region by the cholinomimetic drug carbachol has been shown to generate rhythmic field activity at theta and gamma frequency in vitro (Konopacki et al., 1987, 1988; MacVicar and Tse, 1989; Bland and Colom, 1993a; Huerta and Lisman 1993, 1996; Fisahn et al., 1998), although its relevance to in vivo theta has been debated (Traub et al., 1992). A major source of this controversy is the difference in the location of theta dipoles in the intact animal and in the slice preparation. The present experiment sheds light on this issue. Partial coherence analysis identified tightly coupled theta oscillations in the CA1 str. radiatum and the inner molecular layer of the dentate gyrus. The CA1 str. radiatum and dentate inner molecular layers are the targets of the associational-commissural afferents, originating from the CA3 pyramidal cells and dentate mossy cells, respectively (cf. Amaral and Witter, 1989). Activation of these inputs by electrical stimulation produces extracellular postsynaptic potentials and current sinks in 


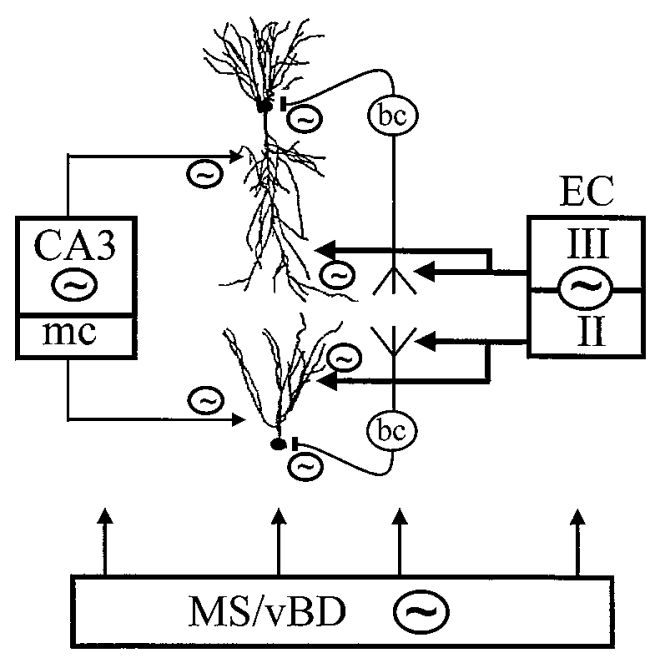

Figure 14. Cooperation of extrahippocampal (septum, entorhinal cortex, $E C)$ and intrahippocampal (CA3, mossy cells, $m c$ ) theta generators. The septal pacemaker [medial septum/vertical limb of diagonal band (MS/ $v B D)$ ] may affect each member of the circuitry and is a requisite of theta oscillation. The main current generator of extracellular theta is the entorhinal input from layers II and III. The rhythmic entorhinal input discharges basket $(b c)$ and chandelier cells (data not shown) and depolarizes pyramidal and granule cells. In the intact hippocampus, the intrahippocampal theta generator $(\mathrm{CA} 3, m c)$ is suppressed by the inhibitory output of the dentate gyrus (data not shown). Only a few CA3 and mossy cells are selectively active. Coherent and converging activity of the entorhinal and intrahippocampal association inputs are critical for the timing of discharges of CA1 pyramidal and granule cells during the theta cycle.

the CA1 radiatum (Andersen, 1960) and dentate inner molecular layer (Deadwyler et al., 1975; Berger et al., 1981; Buzsáki et al., 1986; Brankack et al., 1993; Bragin et al., 1995; Wu et al., 1998). Theta-associated weak rhythmic sinks have also been observed in CA1 radiatum and dentate inner molecular at a phase different from the large sink at the hippocampal fissure (Brankack et al., 1993). Although the theta-related extracellular currents are small in these layers, the partialization method could clearly identify their presence and their significant coherence.

It is notable in this context that large jumps in theta phase were observed in the inner third of the molecular layer (Buzsáki et al., 1986), suggesting that theta input to this layer is relatively independent from the theta conveyed by the perforant path input to the granule cells. After bilateral lesion of the entorhinal cortex, the activity of the CA3 theta oscillator was enhanced several-fold, whereas the selective high theta coherence between signals recorded from the hippocampal fissure and str. oriens disappeared. Overall, these observations suggest the presence of an intrahippocampal theta oscillator (Fig. 14). Because in the in vitro slice preparation extrahippocampal theta inputs are absent, this intrahippocampal theta generation may be activated under appropriate pharmacological conditions. We suggest that the CA3-mossy cell theta oscillator is tonically inactivated by the dentate gyrus output in the intact brain (Acsády et al., 1998) as indicated by the reciprocal relationship between the magnitude of theta signals in the CA1 str. radiatum and str. lacunosum moleculare.

Although relatively weak, the rhythmic theta input from the CA3 pyramidal cells and mossy cells to their target CA1 pyramidal cells and granule cells may be critical for the physiological operation of the intact hippocampal network (Lisman, 1999). The output of a small number of active (place) cells of the CA3 region may condition a large group of CA1 pyramidal cells, some of which may be concurrently excited by the direct perforant path input. The relative magnitude and phase relationship between these extrahippocampal (perforant path) and intrahippocampal (CA3) inputs, in turn, may be responsible for the timing of the action potentials of CA1 pyramidal cells during the theta cycle (O’Keefe and Recce, 1993; Skaggs and McNaughton, 1996).

\section{REFERENCES}

Acsády L, Kamondi A, Sik A, Buzsáki G (1998) GABAergic cells are the major postsynaptic targets of mossy fibers in the rat hippocampus. J Neurosci 18:3386-3403.

Alonso A, Garcia-Austt E (1987) Neuronal sources of the theta rhythm in the entorhinal cortex of the rat. II. Phase relations between unit discharges and theta field potentials. Exp Brain Res 67:502-509.

Amaral DG, Witter MP (1989) The three-dimensional organization of the hippocampal formation: a review of anatomical data. Neuroscience 31:571-591.

Andersen P (1960) Interhippocampal impulses. II. Apical dendritic activation of CA1 neurons. Acta Physiol Scand 48:178-208.

Apostol G, Creutzfeldt OD (1974) Crosscorrelation between the activity of septal units and hippocampal EEG during arousal. Brain Res 67:65-75.

Artemenko DP (1972) Role of hippocampal neurons in theta-wave generation. Neurophysiology 4:409-415.

Barnes CA (1990) Effects of aging on the dynamics of information processing and synaptic weight changes in the mammalian hippocampus. Prog Brain Res 86:89-104.

Barrenechea C, Pedemonte M, Nunez A, Garcia-Austt E (1995) In vivo intracellular recordings of medial septal and diagonal band of Broca neurons: relationships with theta rhythm. Exp Brain Res 103:31-40.

Berger TW, Semple-Rowland S, Bassett JL (1981) Hippocampal polymorph neurons are the cells of origin for ipsilateral activation and commissural afferents to the dentate gyrus. Brain Res 215:329-336.

Bland BH (1986) Physiology and pharmacology of hippocampal formation theta rhythms. Prog Neurobiol 26:1-54.

Bland BH, Colom LV (1993a) Extrinsic and intrinsic properties underlying oscillation and synchrony in limbic cortex. Prog Neurobiol 41:157-208.

Bland BH, Colom LV (1993b) Extrinsic and intrinsic properties underlying oscillation and synchrony in limbic system. Prog Neurobiol 41:157-208.

Bland BH, Andersen P, Ganes T (1975) Two generators of hippocampal theta activity in rabbits. Brain Res 94:199-218.

Bragin A, Jando G, Nadasdy Z, Hetke J, Wise K, Buzsáki G (1995) Gamma $(40-100 \mathrm{~Hz})$ oscillation in the hippocampus of the behaving rat. J Neurosci 15:47-60.

Brankack J, Stewart M, Fox SE (1993) Current source density analysis of the hippocampal theta rhythm: associated sustained potentials and candidate synaptic generators. Brain Res 615:310-327.

Brazhnik ES, Fox SE (1997) Intracellular recordings from medial septal neurons during hippocampal theta rhythm. Exp Brain Res 114:442-453.

Buhl EH, Han ZS, Lorinczi Z, Stezhka VV, Karnup SV, Somogyi P (1994) Physiological properties of anatomically identified axo-axonic cells in the rat hippocampus. J Neurophysiol 71:1289-1307.

Bullock TH, Buzsáki G, McClune MC (1990) Coherence of compound field potentials reveals discontinuities in the CA1-subiculum of the hippocampus in freely moving rats. Neuroscience 38:609-619.

Buzsáki G (1989) A two-stage model model of memory trace formation: a role of "noisy" brain states. Neuroscience 31:551-570.

Buzsáki G, Eidelberg E (1982) Direct afferent excitation and long-term potentiation of hippocampal interneurons. J Neurophysiol 48:597-607.

Buzsáki G, Leung LS, Vanderwolf CH (1983) Cellular basis of hippocampal EEG in the behaving rat. Brain Res Rev 6:139-171.

Buzsáki G, Czopf J, Kondakor I, LK (1986) Laminar distribution of hippocampal rhythmic slow activity (RSA) in the behaving rat: current source density analysis. Brain Res 365:125-137.

Buzsáki G, Bickford RG, Ryan LJ, Young S, Prohaska O, Mandel RJ, Gage FH (1989) Multisite recording of brain field potentials and unit activity in freely moving rats. J Neurosci Methods 28:209-217.

Buzsáki G, Horvath Z, Urioste R, Hetke J, Wise K (1992) High frequency network oscillation in the hippocampus. Science 256:1025-1027.

Buzsáki G, Ylinen A, Penttonen M, Bragin A, Nadasdy Z, Chrobak JJ 
(1995) Possible physiological role of the perforant path-CA1 projection. Hippocampus 5:141-146.

Chrobak JJ, Buzsáki G (1998) Gamma oscillations in the entorhinal cortex of the freely behaving rat. J Neurosci 18:388-398.

Cobb SR, Buhl EH, Halasy K, Somogyi P (1995) Synchronization of Neuronal activity in hippocampus by individual GABAergic interneurons. Nature 378:75-98.

Colbert CM, Levy WB (1992) Electrophysiological and pharmacological characterization of perforant path synapses in CA1: mediation by glutamate receptors. J Neurophysiol 68:1-8.

Csicsvari J, Hirase H, Czurkó A, Mamiya A, Buzsáki G (1999) Oscillatory coupling of hippocampal pyramidal cells and interneurons in the behaving rat. J Neurosci 19:274-287.

Deadwyler SA, West JR, Cotman CW, Lynch GS (1975) A neurophysiological analysis of the commissural projections to the dentate gyrus of the rat. J Neurophysiol 38:164-184.

Dumermuth G, Molinari L (1991) Relationship among signals, cross spectral analysis of the EEG. In: Digital biosignal processing (Weitkunat R,ed), pp 361-398. Amsterdam: Elsevier.

Fisahn A, Pike FG, Buhl EH, Paulsen O (1998) Cholinergic induction of network oscillations at $40 \mathrm{~Hz}$ in the hippocampus in vitro. Nature 394:186-189.

Fox SE (1989) Membrane potential and impedance changes in hippocampal pyramidal cells during theta rhythm. Exp Brain Res 77:283-294.

Fox SE, Wolfson S, Ranck JBJ (1986) Hippocampal theta rhythm and the firing of neurons in walking and urethane anesthetized rats. Exp Brain Res 62:495-508.

Freund TF, Antal M (1988) GABA-containing neurons in the septum control inhibitory interneurons in the hippocampus. Nature 336:170-173.

Fujita Y, Sato T (1964) Intracellular records from hippocampal pyramidal cells in rabbit during theta rhythmic activity. J Neurophysiol 27:1011-1025.

Gaztelu JM, Buno WJ (1982) Septo-hippocampal relationships during EEG theta rhythm. Electroencephalogr Clin Neurophysiol 54:375-387.

Gersch W, Goddard GV (1970) Epileptic focus location: spectral analysis method. Science 169:701-702.

Gogolak G, Stumpf C, Petsche H, Sterc J (1968) The firing pattern of septal neurons and the form of the hippocampal theta wave. Brain Res 7:201-207.

Hasselmo ME, Bower JM (1993) Acetylcholine and memory. Trends Neurosci 16:218-222.

Holscher C, Anwyl R, Rowan MJ (1997) Stimulation on the positive phase of hippocampal theta rhythm induces long-term potentiation that can be depotentiated by stimulation on the negative phase in area CA1 in vivo. J Neurosci 16:6470-6477.

Huerta PT, Lisman JE (1993) Heightened synaptic plasticity of hippocampal CA1 neurons during a cholinergically induced rhythmic state. Nature 364:723-725.

Huerta PT, Lisman JE (1996) Bidirectional synaptic plasticity induced by a single burst during cholinergic theta oscillation in the CA1 in vitro. Neuron 15:1053-1063.

Jenkins GM, Watts DG (1968) Spectral analysis and its applications. San Francisco: Holden-Day.

Kaminski M, Blinowska K, Szelenberger W (1995) Investigation of coherence structure and EEG activity propagation during sleep. Acta Neurobiol Exp 55:213-219.

Kaminski M, Blinowska K, Szelenberger W (1997) Topographic analysis of coherence and propagation of EEG activity during sleep and wakefulness. Electroencephalogr Clin Neurophysiol 102:216-227.

Kamondi A, Acsády L, Buzsáki G (1998a) Dendritic spikes are enhanced by cooperative network activity in the intact hippocampus. J Neurosci 18:3919-3928.

Kamondi A, Acsády L, Wang X-J, Buzsáki G (1998b) Theta oscillations in somata and dendrites of hippocampal pyramidal cells in vivo: activity-dependent phase-precession of action potentials. Hippocampus $8: 244-261$.

King C, Recce M, O'Keefe J (1998) The rhythmicity of cells of the medial septum/diagonal band of Broca in the awake freely moving rat: relationships with behaviour and hippocampal theta. Eur J Neurosci 10:464-477.

Kiss J, Buzsáki G, Morrow JS, Glantz SB, Leranth C (1996) Entorhinal cortical innervation of parvalbumin-containing neurons (basket and chandelier cells) in the rat Ammon's horn. Hippocampus 6:239-246.
Kocsis B (1994) Basis for differential coupling between rhythmic discharges of sympathetic efferent nerves. Am J Physiol 267:R1008-R1019.

Kocsis B, Vertes RP (1994) Characterization of neurons of the supramammillary nucleus and mammillary body that discharge rhythmically with the hippocampal theta rhythm in the rat. J Neurosci 14:7040-7052.

Kocsis B, Vertes RP (1997) Phase relations of theta rhythmic firing of neurons in the supramammillary nucleus and mammillary body to the hippocampal theta activity in urethane anesthetized rats. Hippocampus 7:204-214.

Kocsis B, Gebber GL, Barman SM, Kenney MJ (1990) Relationships between activity of sympathetic nerve pairs: phase and coherence. Am J Physiol 259:R549-R560.

Kocsis B, Thinschmidt JS, Kinney GG, Vertes RP (1994) Separation of the hippocampal theta rhythm generators by partial coherence analysis in the rat. Brain Res 660:341-345.

Konopacki J, MacIver MB, Bland BH, Roth SH (1987) Carbacholinduced EEG "theta" activity in hippocampal brain slices. Brain Res 405:196-198.

Konopacki J, Bland BH, Roth SH (1988) Carbachol-induced theta in hippocampal formation slices: evidence for a third generator of theta in CA3C area. Brain Res 451:33-42.

Konopacki J, Bland BH, Colom LV, Oddie SD (1992) In vivo intracellular correlates of hippocampal formation theta-on and theta-off cells. Brain Res 586:247-255.

Korzeniewska A, Kasicki S, Kaminski M, Blinowska KJ (1997) Information flow between hippocampus and related structures during various types of rat's behavior. J Neurosci Methods 73:49-60.

Larson J, Lynch G (1986) Induction of synaptic potentiation in hippocampus by patterned stimulation involves two events. Science 232:985-988.

Lee MG, Chrobak JJ, Sik A, Wiley RG, Buzsáki G (1994) Hippocampal theta activity following selective lesion of the septal cholinergic system. Neuroscience 62:1033-1047.

Leung LS (1984) Model of gradual phase shift of theta rhythm in the rat. J Neurophysiol 52:1051-1065.

Leung LS, Yim CY (1986) Intracellular records of theta rhythm in hippocampal CA1 cells of the rat. Brain Res 367:323-327.

Leung LS, Yim CY (1991) Intrinsic membrane potential oscillations in hippocampal neurons in vitro. Brain Res 553:261-274.

Li X-G, Somogyi P, Tepper JM, Buzsáki G (1992) Axonal and dendritic arborization of an intracellularly labeled chandelier cell in the CA1 region of rat hippocampus. Exp Brain Res 90:519-525.

Liberati D, Cursi M, Locatelli T, Comi G, Cerutti S (1997) Total and partial coherence analysis of spontaneous and evoked EEG by means of multi-variable autoregressive processing. Med Biol Eng Comput 35:124-130.

Lisman JE (1999) Relating hippocampal circuitry to function: Recall of memory sequences by reciprocal dentate-CA3 interactions. Neuron 22:233-242.

Lopes da Silva FH, Vos JE, Mooibroek J, van Rotterdam A (1980a) Partial coherence analysis of thalamic and cortical alpha rhythms in dog: a contribution towards a general model of the cortical organization of rhythmic activity. In: Rhythmic EEG activities and cortical functioning (Pfurtscheller G, ed), pp 33-59. Amsterdam: Elsevier.

Lopes da Silva FH, Vos JE, Mooibroek J, van Rotterdam A (1980b) Relative contributions of intracortical and thalamo-cortical processes in the generation of alpha rhythms, revealed by partial coherence analysis. Electroencephalogr Clin Neurophysiol 50:449-456.

Lopes da Silva FH, Witter M, Boejinga PH, Lohman A (1990) Anatomic organization and physiology of the limbic cortex. Physiol Rev 70:453-511.

MacVicar BA, Tse FWY (1989) Local neuronal circuitry underlying cholinergic rhythmical slow activity in CA3 area of rat hippocampal slices. J Physiol (Lond) 417:197-212.

McMahon LL, Williams JH, Kauer JA (1998) Functionally distinct groups of interneurons identified during rhythmic carbachol oscillations in hippocampus in vitro. J Neurosci 18:5640-5651.

Mitchell SJ, Ranck JBJ, Steward O, Olton DS (1982) Medial septal area lesions disrupt theta rhythm and cholinergic staining in medial entorhinal cortex and produce impaired radial arm maze behavior in rats. J Neurosci 2:292-302.

Nunez A, Garcia-Austt E, Buno WJ (1987) Intracellular theta rhythm generation in identified hippocampal pyramids. Brain Res 416:289-300.

Nunez A, Garcia-Austt E, Buno WJ (1990) Synaptic contributions to 
theta rhythm genesis in rat CA1-CA3 hippocampal pyramidal neurons in vivo. Brain Res 533:176-179.

O'Keefe J, Recce ML (1993) Phase relationship between hippocampal place units and the EEG theta rhythm. Hippocampus 3:317-330.

Petsche H, Stumpf CH, Gogolak G (1962) The significance of the rabbit's septum as a relay station between the midbrain and the hippocampus. I. The control of hippocampus arousal activity by the septum cells. Electroencephalogr Clin Neurophysiol 14:202-211.

Sekihara K, Abraham-Fuchs K, Stefan H, Hellstrandt E (1996) Suppression of background brain activity influence in localizing epileptic spike sources from biomagnetic measurements. Brain Topogr 8:323-328.

Sherman DL, Tsai YC, Rossell LA, Mirski MA (1997) Spectral analysis of a thalamus-to-cortex seizure pathway. IEEE Trans Biomed Engn 44:657-664.

Skaggs WE, McNaughton BL (1996) Replay of neuronal firing sequences in rat hippocampus during sleep following spatial experience. Science 271:1870-1873.

Skaggs WE, McNaughton BL, Wilson MA, Barnes CA (1996) Theta phase precession in hippocampal neuronal populations and the compression of temporal sequences. Hippocampus 6:149-173.

Soltesz I, Deschenes M (1993) Low- and high-frequency membrane potential oscillations during theta activity in CA1 and CA3 pyramidal neurons of the rat hippocampus under ketamine-xylazine anesthesia. J Neurophysiol 70:97-116.

Stewart M, Fox SE (1989) Firing relations of medial septal neurons to the hippocampal theta rhythm in urethane anesthetized rats. Exp Brain Res 77:507-516.

Stewart M, Fox SE (1990) Do septal neurons pace the hippocampal theta rhythm? Trends Neurosci 13:163-168.

Toth K, Freund TF, Miles R (1997) Disinhibition of rat hippocampal pyramidal cells by GABAergic afferents from the septum. J Physiol (Lond) 500:463-474.

Traub RD, Miles R, Buzsáki G (1992) Computer simulation of carbachol-driven rhythmic population oscillations in the CA3 region of the in vitro hippocampus. J Physiol (Lond) 451:653-672.

Tucker DM, Roth DL, Bair TB (1986) Functional connections among cortical regions: topography of EEG coherence. Electroencephalogr Clin Neurophysiol 63:242-250.

Vanderwolf CH (1969) Hippocampal electrical activity and voluntary movement in the rat. Electroencephalogr Clin Neurophysiol 26:407-418.

Vertes RP, Kocsis B (1997) Brainstem-diencephalo-septohippocampal systems controlling the theta rhythm of the hippocampus. Neuroscience 81:893-926.

Vinogradova OS (1995) Expression, control, and probable functional significance of the neuronal theta rhythm. Prog Neurobiol 45:523-583.

Wallenstein GV, Eichenbaum H, Hasselmo ME (1998) The hippocampus as an associator of discontiguous events. Trends Neurosci 21:317-323.

Williams JH, Kauer JA (1997) Properties of carbachol-induced oscillatory activity in rat hippocampus. J Neurophysiol 78:2631-2640.

Winson J (1974) Patterns of hippocampal theta rhythm in the freely moving rat. Electroencephalogr Clin Neurophysiol 36:291-301.

Wu K, Canning KJ, Leung LS (1998) Functional interconnections between CA3 and dentate gyrus revealed by current source density analysis. Hippocampus 8:217-230.

Ylinen A, Soltesz I, Bragin A, Penttonen M, Sik A, Buzsáki G (1995) Intracellular correlates of hippocampal theta rhythm in identified pyramidal cells, granule cells and basket cells. Hippocampus 5:78-90. 